ANL/TM- -484

DE91 002956

\title{
THE ADVANCED SOFTWARE DEVELOPMENT AND COMMERCIALIZATION PROJECT ${ }^{1}$ Progress Report PR-1
}

\author{
T. R. Canfield, M. Minkoff, C. Mueller, E. Plaskacz, D. P. Weber, \\ D. M. Anderson, I. U. Therios \\ Computing and Telecommunications Division \\ Argonne National Laboratory \\ 9700 South Cass Ave., Argonne, IL 60439-4844 \\ S. Aslam, R. Bramley, H.-C. Chen, G. Cybenko, \\ E. Gallopoulos, H. Gao, A. Malony and A. Sameh \\ Center for Supercomputing Research and Development \\ University of Illinois at Urbana. Champaign \\ Urbana, Illinois 61801-2932 \\ E. Gallopoulos, editor
}

September 1990

\section{MASTER}

\footnotetext{
${ }^{1}$ Work supported by the State of Illinois Technology Challenge Grant, Grant No. 90-82144 with additional support from the National Science Foundation Grant No. CCR900000N for the use of the Cray X-MP/48 at the National Cenier for Supercomputing Applications, University of Ilinois at Urbana.Champaign.
} 


\section{Contents}

1 Introduction 1

1.1 Summary progress for first phase $\ldots \ldots \ldots \ldots \ldots \ldots$

1.2 Acknowledgments ........................ 2

2 Computational Environment 3

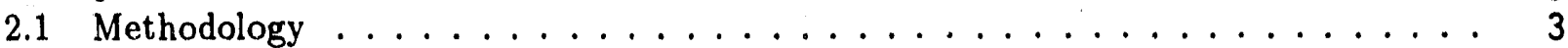

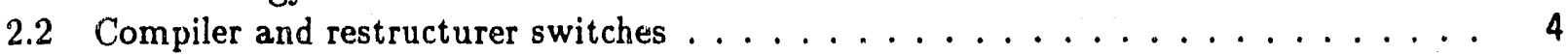

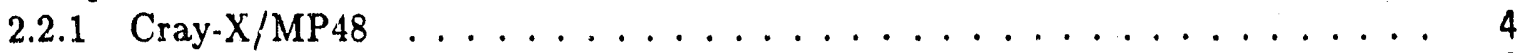

2.2 .2 Alliant $\mathrm{FX} / 8[0] \ldots \ldots \ldots \ldots \ldots \ldots \ldots \ldots$

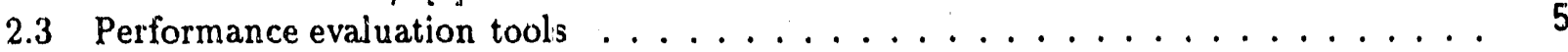

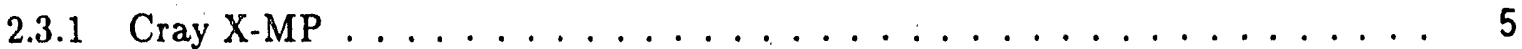

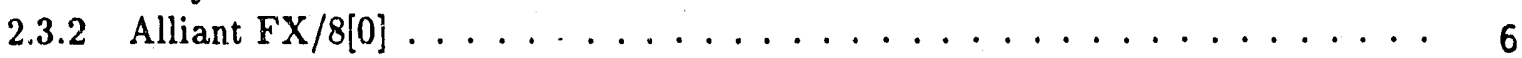

3 COMMIX code $\quad 7$

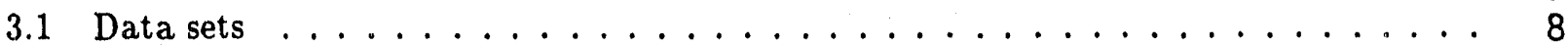

3.2 Results from COMMIX $1 \mathrm{AR} / \mathrm{P} \ldots \ldots \ldots \ldots \ldots$

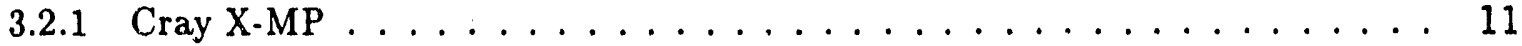

3.2.1.1 Summary of baseline runs . . . . . . . . . . . . . 12

3.2.1.2 Enhanced optimization options and further results . . . . . . 12

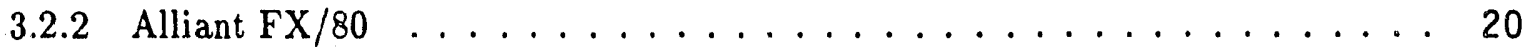

3.2.3 Dynamic program execution tracing analysis $\ldots \ldots \ldots \ldots$

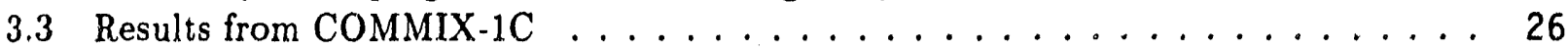

4 WHAMS-3D description 31

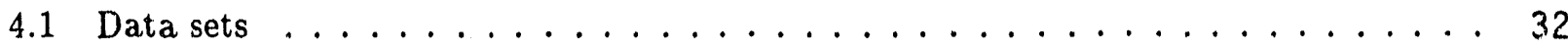

4.2 Results from WHAMS 3 D $\ldots \ldots \ldots \ldots \ldots \ldots \ldots$

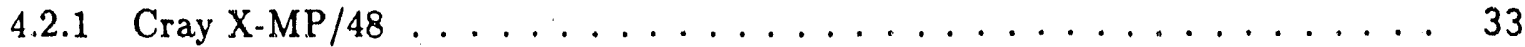

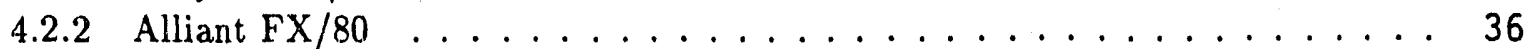

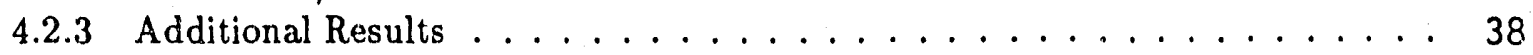

4.2 .4 Dynamic program execution tracing analysis ............ 40

5 Conclusion $\quad 42$

Bibliography $\quad 43$

A Appendix: Milestones for FY 1991 


\section{List of Tables}

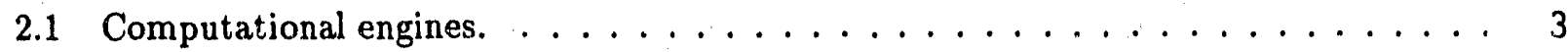

3.1 Execution tines for SV, SV(Zv) and SVC COMMIX-1AR/P on the Cray X-MP/48

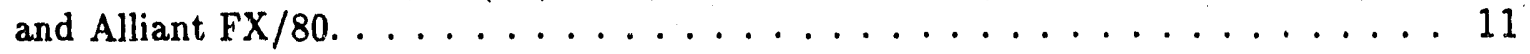

3.2 Execution times and MFLOPS for COMMIX-1AR/P baseline SV performance on Cray X-MP/14 (from н $\mathrm{PM}) . \ldots \ldots \ldots \ldots \ldots$

3.3 Characteristics of most time-consuming subroutines of COMMIX-1AR/P for data set $\mathrm{P} 1 \mathrm{r} 0$ running in baseline SV mode on Cray X.MP (from PERFTRACE). . . . . 13

3.4 Characteristics of most time-consuming subroutines of COMMIX-1AR/P for data set P1r1 running in baseline SV mode on Cray X-MP (from PERFTRACE). . . . . 13

3.5 Characteristics of most time-consuming subroutines for data set P1r2 running in baseline SV mode on Cray X-MP (from PERFTrACE). . . . . . . . . . . . 13

3.6 Characteristics of most time-consuming subroutines for data set P2 running in baseline SV mode on Cray X-MP (from PERFTRACE). . . . . . . . . . . . . 14

3.7 Characteristics of most time-consuming subroutines for data set P3 running in baseline SV mode on Cray X-MP (from PERFTrACE). . . . . . . . . . . . . 14

3.8 HPM group 0 summary for baseline SV COMMIX-1AR/P code running on Cray $\mathrm{X}-\mathrm{MP} / 14$ with data sets $\mathrm{P} 1, \mathrm{P} 2$ and $\mathrm{P} 3 \ldots \ldots \ldots \ldots \ldots$

3.9 нРM group 1 summary for baseline SV COMMIX-1AR/P code running on Cray $\mathrm{X}-\mathrm{MP} / 14$ with data sets $\mathrm{P} 1, \mathrm{P} 2$ and $\mathrm{P} 3 . \ldots \ldots \ldots \ldots$

3.10 н $\mathrm{PM}$ group 2 summary for baseline SV COMMIX-1AR/P code running on Cray $\mathrm{X}-\mathrm{MP} / 14$ with data sets $\mathrm{P} 1, \mathrm{P} 2$ and $\mathrm{P} 3 . \ldots \ldots \ldots \ldots \ldots$

3.11 HPM group 3 summary for baseline SV COMMIX-1AR/P code running on Cray $\mathrm{X}-\mathrm{MP} / 14$ with data set P1. .................. 15

3.12 нPM group 3 summary for baseline SV COMMIX-1AR/P code running on Cray $\mathrm{X}-\mathrm{MP} / 14$ with data sets $\mathrm{P} 2$ and $\mathrm{P} 3 . \ldots \ldots \ldots \ldots \ldots$

3.13 Execution times and MFLOPS for SV(Zv) COMMIX-1AR/P code running on Cray $\mathrm{X}-\mathrm{MP} / 48$ (from н $\mathrm{PM}) . \ldots \ldots \ldots \ldots \ldots \ldots$

3.14 Timing profile for COMMIX-1AR/P running on Cray X-MP/48, compiled in SV(Zv)

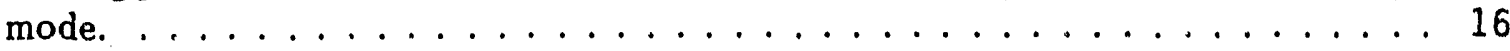

3.15 Profile of the most time consuming subroutines in $S V(Z v)$ mode for COMMIX1 AR/P running on Cray X-MP/48 (from FLowtraCE). . . . . . . . . 17

3.16 MFLOPS profile of the most time consuming subroutines in baseline SV, and enhanced vectorization $\mathrm{SV}(\mathrm{Zv})$ modes for the COMMIX-1AR/P code running on Cray $\mathrm{X}-\mathrm{MP}$ using data decks $\mathrm{P} 1 \mathrm{r} 0$ and $\mathrm{P} 1 \mathrm{r} 2$ (from PERfTrACE). . . . . . . . . 18 
3.17 Execution time profile for the SVC version of COMMIX-1AR/P running on Cray $\mathrm{X}-\mathrm{MP} / 48$ (from H PM).

3.18 н PM group 0 summary $f_{r} \mathrm{SV}(\mathrm{Zv})$ and SVC COMMIX-1AR/P code running on Cray $\mathrm{X}-\mathrm{MP} / 48$ with data set P1.

3.19 Ratio of scalar and vector floating operations for COMMIX-1AR/P code running on Cray X-MP/48 with data set P1.

3.20 H PM group 1 summary for $S V(Z v)$ and SVC COMMIX-1AR/P code running on Cray

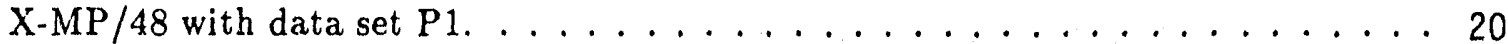

3.21 HPM group 2 summary for $\mathrm{SV}(\mathrm{Zv})$ and SVC COMMIX-1AR/P code running on 4-CPU Cray X-MP/48 with data set P1. . . . . . . . . . . . . . . . 21

3.22 H PM group 3 summary for SV(Zv) COMMIX-1AR/P code running on Cray X-MP/48 with data set P1. . . . . . . . . . . . . . . . . . . . 21

3.23 HPM group 3 summary for SVC COMMIX-1AR/P code running on Cray X-MP/48

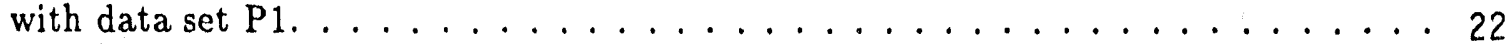

3.24 Execution times for SV, SC and SVC COMMIX-1AR/P code running on an Alliant

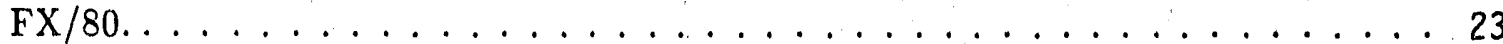

3.25 Effect of number of CEs on execution times for SVC COMMIX-1AR/P code running

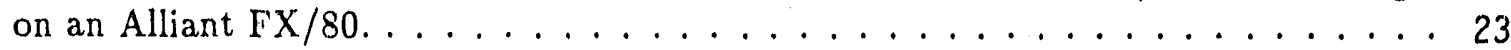

3.26 Execution times of various phases for COMMIX-1AR/P code running on an Alliant $\mathrm{FX} / 80$ for P1r0.

3.27 Execution times of CPU intensive routines in COMMIX-1AR/P code running on an Alliant FX/80 for P1r0.

3.28 Execution times of various phases for COMMIX-1AR/P code running on an Alliant FX/80 for P1r1.

3.29 Execution times of CPU intensive routines in COMMIX-1AR/P code running on an Alliant FX/80 for P1r1.

3.30 Execution times of various phases for COMMIX-1AR/P code running on an Alliant $\mathrm{FX} / 80$ for P1r2.

3.31 Execution times of CPU intensive routines in COMMIX-1AR/P code running on an Alliant FX/80 ior P1r2.

3.32 Routine events for COMMIX-1AR/P trace graph obtained from the Cray X-MP/48. 26

3.33 Timing results from COMMIX-1C runs on the Sparc, Cray X-MP/14 and Alliant $\mathrm{FX} / 8$.

3.34 H PM group 0 summary for baseline SV COMMIX-1C code running on Cray X-MP/14 with data set C1r2.

3.35 HPM group 1 summary for baseline SV COMMIX-1C code running on Cray X-MP/14

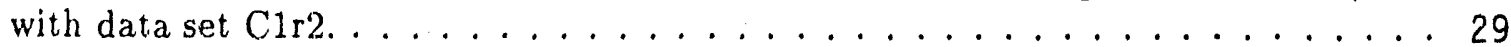

3.36 H PM group 2 summary for baseline SV COMMIX-1C code running on Cray X-MP/14 with data set $\mathrm{C} 1 \mathrm{r} 2 \ldots \ldots \ldots \ldots \ldots \ldots$

3.37 HPM group 3 summary for baseline SV COMMIX-1C code running on Cray X-MP/14 with data set $\mathrm{C} 1 \mathrm{r} 2 \ldots \ldots \ldots \ldots \ldots \ldots \ldots \ldots$

4.1 Execution times for WHAMS-3D on the Cray X-MP/48 (from HPM). . . . . . . 33

4.2 Execution times for SVC version of WHAMS-3D on the Cray X-MP/48 (from HPM).

4.3 Floating point operations on Cray X-MP/48 for the $S$ version (from group 0 of $\mathrm{HPM}$ ). 
4.5 Performance data for WHAMS-3D on the Cray X-MP/48 using data set cylpanel

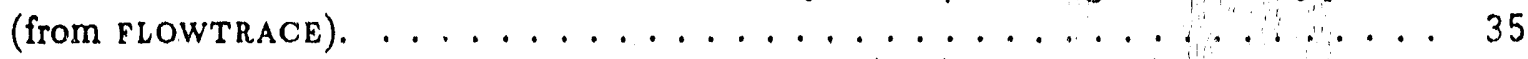

4.6 Execution time for WHAMS-3D on the Alliant FX/80 (from GProf). . . . . 36

4.7 Execution time for the SC version of WHAMS-3D on the Alliant XX/80 (from

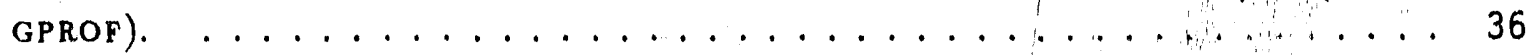

4.8 Execution times for the SVC version of WHAMS-3D on the Alliant $\mathrm{FX} / 80$ (from

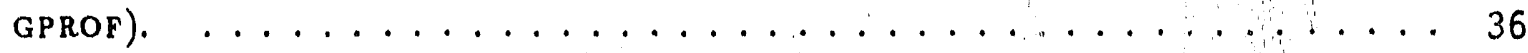

4.9 MFLOPS for WHAMS-3D on the Alliant FX/80 . . . . . . . . . . 37

4.10 Performance data for WHAMS-3D on the Alliant FX/80 using data set cylpanel

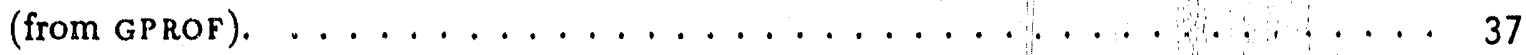

4.11 Characteristics of the data sets used in WHAMS-3D. . . . . . . . . . 38

4.12 Execution times for WHAMS-3D on the ANL Alliant FX/8. . . . . . . 38

4.13 Execution times for WHA.MS-3D on the Sparc, Cray X-MP/14, and Alliant FX/8. $\quad 39$

4.14 H PM performance data summary for WHAMS-3D running on the Cray X-MP/14. . 39

4.15 Routine events for WHAMS-3D trace graph . . . . . . . . . . . 40 


\section{Chapter 1}

\section{Introduction}

This is the first of a series of reports pertaining to progress in the Advanced Software Development and Comniercialization Project, a joint collaborative effort between the Center for Supercomputing Research and Development of the University of Illinois and the Computing and Telecommunications Division of Argonne National Laboratory.

The purpose of this work is to apply techniques of parallel computing that were pioneered by University of Illinois researchers to mature computational fluid dynamics (CFD) and structural dynamics (SD) computer codes developed at Argonne.

The collaboration in this project will bring this unique combination of expertise to bear, for the first time, on industrially important problems. By so doing, it will expose the strengths and weaknesses of existing techniques for parallelizing programs and will identify those problems that, need to be solved in order to enable wide spread production use of parallel computers. Secondly, the increased efficiency of the CFD and SD codes themselves will enable the simulation of larger, more accurate engineering models that involve fluid and structural dynamics. Such an enhanced capability is fundamental to industrial efficiency and competitiveness, and could serve as an exemplary model for similar future activities.

In order to realize the above two goals, we are considering two production codes that have been developed at ANL and are widely used by both industry and Universities. These are COMMIX and WHAMS-3D. The first is a computational fluid dynamics code that is used for both nuclear reactor design and safety and as a design tool for the casting industry. The second is a three-dimensional structural djnamics code used in nuclear reactor safety as well as crashworthiness studies. These codes are currently available for both sequential and vector computers only. Our main goal is to port and optimize these two codes on shared memory unultiprocessors. In so doing, we shall establish a process that can be followed in optimizing other sequential or vector engineering codes for parallel processors.

\subsection{Summary progress for first phase}

We have completed the first phase of tasks and deliverables specified in the work plan shown in Appendix A, as copied from the Proposal of the Aclvanced Software Development and Commercialization Project funded by the State of Illinois Technology Challenge Grant, Grant No. 90-82144.

We summarize our accomplishments here.

1. The codes have been ported to the target multiprocessor machines (Cray X-MP/48 at NCSA, 
Alliant $\mathrm{FX} / 8(0)$ at $\mathrm{CSRD}$ and $\mathrm{ANL})$.

2. Appropriate data sets have been selected to test the codes' performance. These fully exercise all aspects of the codes, in anticipation for the industrial data sets to be obtained in the context of close cooperation with Illinois industry (see below).

3. It was stated from the beginning that our main objective is to demonstrate the effectiveness of our techniques for problems of industrial application. We have established collaboration with Commonwealth Edison and the Thermal Hydraulics Section of their Nuclear Fuel Services Department has given to ANL an important industrial data set for one of our CFD codes (COMMIX 1AR/P). This problem requires a 12 hour simulation when performed on one vector processor of Commonwealth Edison's IBM 3090. Discussions are underway with General Motors, Inland Steel and other companies to secure inputs and collaboration for the structural dynamics code.

4. The codes have been profiled for the first time, their baseline (unoptimized) performance on each of the target machines was recorded, and the most time consuming subroutines have been identified.

5. We have already started applying automatic restructuring tools on some of the codes.

Overall the first phase has sh. Nn that significant improvements in the codes' performance result from vectorization. This is partly because of the effectiveness of vectorizing compilers, and partly because the principles of vectorization have been available to programmers for over 15 years. The first phase also shows that applying existing automatic restructurers for multiprocessing gives little improvement or even degradation in performance, but provides clues on how to achieve better results. This emphasizes the need to move the highly successful tools and techniques for multiprocessing from our research centers to the industrial base.

\subsection{Acknowledgments}

The authors would like to thank R. N. Blomquist, H. M. Domanus, E. M. Gelbard and J. M. Kennedy from Argonne National Laboratory, T. Belytschko from Northwestern Uriv., and $M$. Berry from CSRD for their help during this project. 


\section{Chapter 2}

\section{Computational Environment}

As described earlier, our work is oriented towards standard and mini supercomputers with multiprocessing capabilities. By all accounts these are the machines which are able to sustain the performance requirements for production runs with the complex codes we are dealing with.

For this phase of the experiments we have used the Cray and Alliant machines shown in Table 2.1. In some cases we also report results from runs performed on Sun Sparcs at ANL, due to the natural interest in understanding the performance on what is very likely to be on a scientist's desk, namely a fast workstation used for code development.

A planned addition to the machines of Table 2.1 is the CSRD Alliant FX/2800, whose 28 processors based upon the 8660 RISC chip will be an interesting case study of parallelism and software emulated vector processing.

\subsection{Methodology}

Our overall strategy in these experiments has been the following: We first compile and link on the target machine. If baseline performance is needed we run the code to obtain baseline timing and validate runs. We then compile under different options described in Section 2.2 to test the effect of automatic parallelization and vectorization. The performance for each run was analyzed with the tools described in Section 2.3.

Table 2.1: Computational engines.

\begin{tabular}{||l|l|r|r||}
\hline Machine & $O / S$ & Memory & Location \\
\hline CRAY X-MP/14 & Unicos & $4 \mathrm{MW}$ & ANL \\
CR.AY X-MP/48 & Unicos & $8 \mathrm{MW}$ & NCSA \\
ALLLANT FX/80 & Concentrix/Xylem & $80 \mathrm{MB}$ & CSRD \\
ALLIANT FX/8 & Concentrix & $64 \mathrm{MB}$ & ANL \\
\hline
\end{tabular}




\subsection{Compiler and restructurer switches}

In this section we provide a summary of some of the compilation options used when performing the experiments. We note that since most of these options at some stage invoke source-to-source restructuring compilers, thus attempting automatic vectorization and parallelization, their use and our comments also belong to Phase 2 of this work.

No hand optimizations were performed.

\subsubsection{Cray-X/MP48}

The following optimization modes were used for our benchmarks.

\begin{tabular}{lll} 
Symbol & Command options & Optimizations \\
\hline S & cft77 -o novector & scalar optimization \\
SV & cf77-c - $\mathrm{c}$ & scalar and vector OR \\
& cft77-o & \\
SV(Zv) & cf77-c-Zv & enhanced scalar and vector \\
SVC & $\operatorname{cf} 77-\mathrm{Zp}$ & scalar, vector, concurrent (autotasking)
\end{tabular}

With no options specified, the compiler attempts vectorization of the innermost loop. The $-\mathrm{Zv}$ option causes the compiler to invoke the dependency analyzer FPP, which attempts more compiicated data dependence analyses and inserts directives for vectorization. Although it also inserts directives for microtasking, it does not interpret thern (see below).

Both the cft77 and cf77 commands invoke the CFT77 compiler; cf77 also includes the load step for creating an executable file. Otherwise, cf77 uses most of the options available with the cf 777 command. The -0 is an optimization option which, by default, implies both scalar optimization and vectorization. When -o is followed by the word novector, vectorization is suppressed and only scalar optimization is performed. The option $-\mathrm{Zp}$ causes the compiler to invoke FPP, which inserts directives for vectorization and microtasking. This automatic detection of and instrumentation for microtasking is called autotasking.

Although no hand optimizations were attempted, there were a few cases, documented in Section 3.2 , where the code contained inline optimization directives for the Cray compiler.

\subsubsection{Alliant FX/8[0]}

The code was compiled with Alliant's Fortran compiler and VAST restructurer, with switches selected from the following:

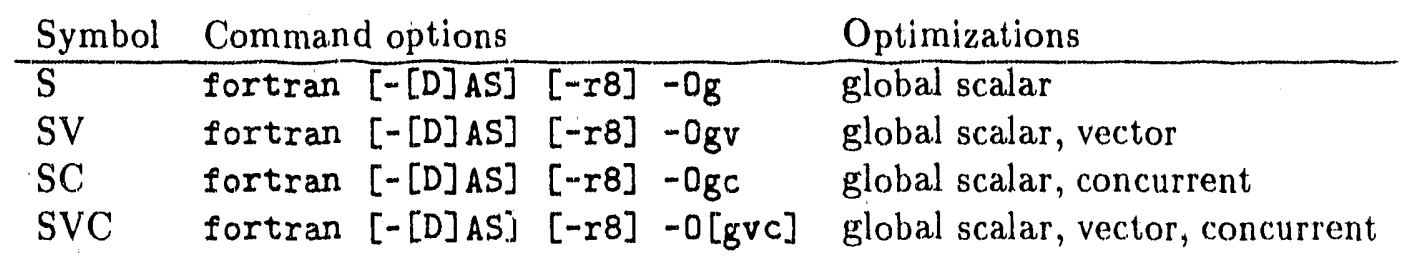

The option - [D]AS means that transformations for optimizing recurrences (based on the currently available number of processors, if the $D$ option is also in effect) can use the associative law of addition. Unless stated otherwise, the use of this option will be assumed.

The option - $r 8$ forces the transformation of all REAL varjables and intrinsics into REAL*8. 
We note that Cray's FPP preprocessor is very similar to Alliant's VAST, as they are both products of Pacific Sierra, purchased by the respective computer companies to restructure code speciftcally for the corresponding machines. Although options and defaults differ, restructuring is done based on essentially the same data dependence information.

\subsection{Performance evaluation tools}

Unless mentioned otherwise, all times are in seconds.

\subsubsection{Cray X-MP}

Performance utilities such as FLOWTRACE, HPM, PERFTRACE on the subroutine level, and PERFTRACE on the loopnest level are available on the Cray X-MP [6]. FLOWTRACE, HPM, and PERFTRACE on the subroutine level were developed by Cray Research, Inc.; PERFTRACE on the loopnest; level for the Cray X-MP/48 was developed by John Larson at NCSA.

FLOWTRACE generates printed information about all procedure calls in a program; its summary contains the following information:

- The time spent in each routines: amount, percentage of the total execution time, and average time per call.

- Number of calls to each procedure.

- Lists of routines that call and are called by each routine.

- A dynamic call tree of the main program and all called subprograms.

HPM reports performance of the entire prol $\mathrm{n}$. It can issue any of four kinds of reports, named groups $0,1,2$ and 3 .

Grour, 0 Scalar activity: number of instructions, memory references, floating-point additions, multiplications, reciprocals, MFLOP.

Group 1 Conditions that delay instruction issue: Percent of time (in clock periods) waiting on resources such as semaphores, shared registers, scalar, vector and address registers functional units, scalar and block memory references.

Group 2 Information on central memory references and conflicts.

Group 3 Instruction types and vector operations.

PERFTRACE gives the same type of statistics about computer performance as those generated by the HPM, but with details for individual program units.

For the Cray X-MP we ran the codes through all four groups of the Hardware Performance Monitor (HPM). We also compiled and linked with each of FLOWTRACE, PERFTRACE and the profiler PROF for routine-by-routine performance monitoring.

As a start of Phase 2 advanced performance analysis, we have generaced some preliminary traces of COMMIX-1AR/P and WHAMS-3D. The goal is to study performance behavior at a more refined level using trace data of routine entry and exit actions. We used tracing tools developed for the Cray X-MP and Cray 2 which are described in [9]. In summary these tools can capture detailed histories of routine invocation together with machine performance statistics. 


\subsubsection{Alliant FX/8[0]}

The tools for performing performance analysis of the codes running on the Alliant $\mathrm{FX} / 8[0]$ were the standard Alliant facility GPROF and calls to the etime() facilities.

The utility GPROF is used to obtain execution profiles of FX/Fortran codes. For each routine, GPROF counts the number of times it was called and determines the time elapsed in its execution. 


\section{Chapter 3}

\section{COMMIX code}

COMMIX is one of the world's premier thermal-hydraulics codes, used at scores of government and industrial sites in support of a vast range of research and development projects. Argonne's development and refinement of COMMIX, which has continued for more than ten years, was originally supported by the U.S. Nuclear Regulatory Commission for application to a wide variety of reactor safety problems. COMMIX has been developed using a unique jorous media approach to the solution of the Navier-Stokes equations in an arbitrary three-dimensioncl region. In its various versions, COMMIX can model separate single-phase fluids, multiphase flows, and free surface flows. The code uses differenced momentum/mass conservation equations which are combined to form a pressure equation. Once the pressures are known, the fluid velocities are updated to provide input to the energy simulation and the next iteration or time step. The hydraulic driving force may be flow or pressure boundary conditions at inlets and outlets, one of several pump models, or a fluid temperature/density distribution. The energy equations are differenced using the updated velocities, and the source terms are accumulated from the treatments of convection boundaries, conduction boundaries, thermal structures, or heat generation in the fluid itself.

One-dimensional shell structures superimposed on the fluid geometry model various fluid sys. tem thermal components such as vessels, pipes, baffles, tube-shell heat exchangers, and reactor fuel. Once the fluid temperature distribution is updated, the submerged thermal structures' internal temperature distributions are recomputed, assuming one-dimensional conduction through each thermal structure segment.

The momentum and fluid energy equation time differencing is implicit, which requires that the difference equation coefficients be constructed from end-of-timestep temperatures and velocities. Since these are not known when the coefficients are computed, a set of "outer" iterations is completed in which the momentum and energy equations' coefficients are computed from ever-better estimates of end-of-step values, a process repeated until the "outers" converge to the end-of-step solution. Each outer iteration consists of pressure matrix equation construction, pressure equation solution, velocity update, energy matrix equation construction, energy equation solution, and, finally, a structure- fluid heat flux update. Only when the time step converges are the radiation heat fluxes between thermal structures updated.

The COMMIX code exists in two versions, COMMIX-1C and COMMIX 1AR/P. The COMMIX family of codes were developed to analyze steady-state/transient, single-phase, three-dimensional compressible/incompressible flow with heat transfer in a reactor system. These codes are also applicable to a broad range of applications. Due to the wide range of applications fundamentally different codes have evolved out of a common software ancestor. We are using COMMIX-1AR/P 
and COMMIX-1C, both of which are the latest derivatives of the COMMIX-1A code and its predecessors. The COMMIX-1A code was designed for thermal-hydraulic analysis of reactor components. It solves the conservation equations of mass, momentum, and energy as a boundary-value differential equation in space and an initial-value problem in time. Spatial discretization is accomplished by a staggered grid system to describe field variables at cell centers and flow variables at cell surfaces. The codes described below represent totally different extensions in both modeling capabilities and targeted computer architectures.

COMMIX-1AR/P is based on COMMIX-1A and contains new models and formulations which were added to the code over a period of about 5 years [5]. These include a pump model, radiation heat transfer, boundary conditions for inlet flow as a function of radiation surface temperature and to simulate expansion ceils fo- constant mass calculations, multiple fluid capability, conjugate gradient solution technique for momentum/mass equations, implicit coupling of thermal structures to fluid, and change-based automatic time step control. It is run primarily on the Cray X-MP/14, and is substantially vectorized.

COMMIX-1C is based on COMMIX-1B [1,2] (and ultimately on COMMIX-1A). It applies three new models to treat turbulence effects including the two-equation $i-\epsilon$ model which has been discretized to simulate subsonic compressible flow. A new porous-medium formation was developed which can be used to model anisotropic flow with stationary structures. The flow-modulated skew-upwind differencing scheme has been implemented to reduce numerically.induced diffusion of scalar transport. Other distinctive options inclucie transient mass flow boundary conditions and application of direct solution of sparse matrix equations. It is run primarily on Sparc workstations at ANL.

\subsection{Data sets}

The data sets used for these experiments are routinely applied by ANL for testing the validity of any modifications to the code. For that reason they are designed to fully exercise the code and are very suitable for the goals of the first phase. We here summarize the results obtained from one data set for COMMIX $1 \mathrm{C}$ and COMMIX $1 \mathrm{AR} / \mathrm{P}$.

\section{$1 \mathrm{AR} / \mathrm{P}$ data sets}

Data set P1 Simulates two-fluid (sodium, air) flow and heat transfer in the 90 degree sector of a generic modular pool-type liquid metal (sodium) reactor. This transient simulates the reactor system's response to a postulate pumping failure in which the pump ramps to zero power linearly over five seconds, and the reactor power ramps to $7 \%$ (initial decay heat) during the first ten seconds. Cylindrical coordinates $(r, \theta, z)$ are used. The computational domain consists of 22 unique surface types, 330 (regular) surface elements and 205 computational cells for a maximum of 8,3 , and 12 cells in the $r, \theta$ and $z$ directions respectively. The data set consists of four input decks corresponding to the following phases:

P1ro Cold Start to Stearly State (1000 time steps);

P1r1 Restart of Steady S :ate (100 time steps);

P1r2 Transient Problem from Steady State (1.100 time steps);

P1r3 Restart of Transient Problem (400 time steps). 
Data set P2 This data set simulates the steady-state behavior of an experiment , serformed at Karlsruhe, Germany, to study the transient behavior of a seven-pin assembly during a flow transient. There are 48 axial meshes, and each pin and its adjacent coolant is represented by four $x-y$ cells. The sodium flows vertically through the pin bundle, which is enclosed in a hexagon steel can. The sodium is heated by the pins as it flows upwards. The transient analyzed (not in this data set) is a linear flowrate decrease, and the event of interest is the time of odium boiling onset. Rectangular geometry is used for 6 unique surface types, 594 surface elements (some of which are irregular), 432 computational cells, for a maximum of 3,3 , and 48 cells in each coordinate direction. There are 4 force structures.

Data set P3 The object of the simulation is to determine in steady-state the degree of thermal relaxation in the Clinch river breeder reactor outlet plenum above the core. The sodium exits reactor subassemblies of three types (driver, blanket, or control), earh with a specific design, power, and flow rate. The mitigation of thermal stress and shock (during transients) depends on the mixing of relatively hotter sodium with the cooler sodium flowing out of the neighboring subassemblies. This issue is also being addressed in the case of state-of-the-art liquid metal cooled reactor designs. The geometry is cylindrical, with 11 unique surfaces, 446 (regular) surface elements, and 346 computational cells, for a maximum of 6,3 , and 23 cells along each coordinate direction.

1C data set This data set was used as input for the 1C version of COMMIX. The TMLB' is one of the postulated reactor accidents that is currently being investigated by the U.S. Nuclear Regulatory Commission. In this accident, several different significant events and physical phenornena occur. During the progression of the TMLB' accident scenario, there is a time when the hot leg dries out and the core becomes uncovered. From that time on, multidimensional natural-circulation phenomena play an important role in heat trainsport and the heat-up of the various components in a reactor system. The multidimensional capabilities of the code make possible the simulation of the natural convection phenomena which are probable in the TMLB' scenario. The generated flow patterns, temperature distributions, and steam generator heat transfer rates provide useful guidance for simulation of one-dimensional systems. This data is needed to support the system analyses being performed at Los Alamos, Sandia and Idaho National Laboratories. In the transient that is simulated, the entire system at time $t=0$ is isothermal, i.e., it contains saturated steam at $p=1.61 \times 10^{7} \mathrm{~Pa}$. For time $t>0$, decay heat was added to the core. While natural convection flow pattern was being established, the system was being perturbed by the opening and closing of the PORV value. The analysis was performed using a PORV model that is at the end of the surge line that is connected to the pressurizer. The geometry is a Cartesian box, with 1606 surface elements and 947 computational cells. The pressure equation is solved by means of the Yale Sparse Matrix Package (YSMP). For this test case, a constant turbulent viscosity model is used. There are three decks of interest:

C1r0 Run to steady state;

C1r1 137 time steps;

C1r2 45 time steps.

Consistent with the goals of this project, to demonstrate the benefits from using multiprocessor architectures on large important codes used by Illinois inclustry, a data set for a real world problem 
has been obtained from the Thermal Hydraulics Section of Commonwealth Edison's Nuclear Fuel Services Department. This data set, which uses COMMIX to verify the conservatism in RETRAN licensing calculations which simulate the reactor's response to a steamline break accident, and takes up to twelve hours of simulation when performed on Commonwealth Edison's IBM 3090 vector processor, will be described in the context of our efforts in future phases of this work.

\subsection{Results from COMMIX 1AR/P}

As a reference point for the $1 \mathrm{AR} / \mathrm{P}$ code we obtained the baseline performance for this data set, meaning that the code was compiled and ran without any automatic optimization options applied to it. The code was originally written so that it runs best on architectures with vector processing. The pressure equations of the mass-momentum loop are solved using a preconditioned conjugate gradient technique, which converges at an acceptable rate without requiring any sensitive iteration parameters from the user. Incomplete Cholesky factorization approximates the matrix inverse, and is mostly vectorized, but with vector lengths frequently far from optimum. No attempt has been made to exploit parallelization here. A large amount of the computing time is spent constructing matrix equations, a process which would require massive recoding to vectorize because of its large, logic-loaded loops. Such loops, however, are expected to lend themselves very well to the parallelization efforts which we plan to pursue in the context of this work.

From the $1 \mathrm{AR} / \mathrm{P}$ data sets we described, we observed that the results obtained when using the decks P1r0 and P1r2 (begin steady state and begin transient) were very similar to those obtained from the restart decks 1 and 3. Moreover, although data sets P2 and P3 are useful for code development purposes, they exercised the code less than P1. Since we did not want to clutter the report with tables, we decided to provide only data from the most interesting and representative experiments. This means that, except for the baseline runs, we usually omit any information from P1r1, P1r3 and P2, P3.

Overall the code consists of six major groups of computations:

1. Momentum-related equations construction.

2. Momentum-related equations solution.

3. Energy-related equations construction.

4. Energy-related equations solution.

5. Thermal structure temperatures computation.

6. Thermal structure radiative heat flux computation.

We next list the function of the most important routines in the set:

ENERGI Construct coefficients in the energy equation.

LOWFCV Solve the upper triangular system as part of the conjugate gradient solution of the pressure equation.

PEQN Construct the coefficients in the pressure equation. 
QSTRDS Calculates finite differences of solid/fluid heat transfer rate over the thermal structures.

QSTRUC Set solid-to-fluid source term for the fluid energy equation.

SOLVEV Solver of linear system for the energy equation using Gauss-Seidel relaxation on red-black ordering.

хмомг, умомг, zмом Sweep over all fluid cells to set-up the $x, y$ and $z$ direction momentum equations.

The original code is instrumented with calls to the Cray intrinsic function second to summarize run times for each of its major steps. Indeed, whenever presenting timing results for the abo'c stages, these results were derived from the original code instrumentation.

As the code was written to take advantage of vector processing in some of its solver routines and in anticipation of the great costs involved otherwise, we decided not to explicitly disable vectorization for the baseline runs.

We note that the only subroutines of the original code containing inline Cray compiler directive lines CDIR\$ were DAXPXC, DAXPYC and LOWFCV. In those routines vectorization was helped using the IVDEP directive, which causes the compiler to ignore vector dependencies in its attempts to vectorize the corresponding Do loops. The effect of this is clearly seen in the performance results presented in Section 3.2.1.

For future reference, we first show Table 3.1, which summarizes the runtimes for each of the machines and compilation options for data sets $\mathrm{P} 1 \mathrm{r} 0$ and $\mathrm{P} 1 \mathrm{r} 2$.

Table 3.1: Execution times for SV, SV(Zv) and SVC COMMIX-1AR/P on the Cray X-MP/48 and Alliant FX/80.

\begin{tabular}{||l|c|l||}
\hline Routine & P1r0 & P1r2 \\
\hline Cray SV & 93.96 & 920.94 \\
Cray SV(Zv) & 81.48 & 872.90 \\
\hline Cray SVC & 225.25 & 2025.48 \\
1. CPU & 22.35 & 297.28 \\
2 CPU & 31.76 & 405.18 \\
3 CPU & 47.19 & 461.88 \\
4 CPU & 123.95 & 861.20 \\
\hline Alliant FX/80 & & \\
SV (1 CE) & $1,322.4$ & $12,331.2$ \\
SC (8 CE) & $1,128.7$ & $10,015.0$ \\
SVC (8 CE) & $1,136.7$ & $10,048.6$ \\
\hline
\end{tabular}

\subsubsection{Cray X-MP}

First we summarize the baseline performance obtained for each of the data sets. As mentioned earlier, the code was written to take take advantage of the vector processing capabilities of a single processor of the Cray X-MP. For the purpose of $1 \mathrm{AR} / \mathrm{P}$ we thus consider as baseline the performance obtained from the code in SV compilation mode (cf. Section 2.2). When appropriate, in the t..'Nes 
of this section, we mention the performance tool used to obtain the results (e.g. PERFTRACE, FLOWTRACE, etc.)

\subsubsection{Summary of baseline runs}

We first report results from the baseline runs (mode SV), as needed to satisfy our milestones for the first phase (cf. Appendix A).

The runtimes for each of the data sets are summarized in Table 3.2.

Table 3.2: Execution times and MFLOPS for COMMIX-1AR/P baseline SV performance on Cray $\mathrm{X}-\mathrm{MP} / 14$ (from нPM).

\begin{tabular}{||l|r|r|r|r|r|r||}
\hline Data set & P1r0 & P1r1 & P1r2 & P1r3 & P2 & P3 \\
\hline Time & 93.96 & 9.44 & 920.94 & 665.10 & 17.84 & 42.24 \\
MFLOPS & 9.12 & 7.93 & 7.68 & 7.14 & 11.43 & 10.02 \\
\hline
\end{tabular}

We next order the most time-consuming subroutines for each of the data sets except P1r3, and report their MFLOPS, the number of times they were called, and the percentage of time (clock periods) spent on each. This data is obtained from PERFTrACE and shown in Tables 3.3-3.7.

\subsubsection{Enhanced optimization options and further results}

As mentioned earlier, by using special (automatic) restructuring options, the results in this section could also be considered as Phase 2 results. Since they were available however, we thought that it is appropriate to report them here.

Table 3.13 summarizes the execution times and MFLOPS for the code under SV(Zv) mode of compilation. We notice an improvement over the baseline times of Table 3.2, implying that the FPP optimizations were effective in certain cases. As will be seen, the greatest effect is seen in the SOLVEV routine.

We show in Table 3.14 the breakdown of times for each phase of the computation, when compiled with the $S V(Z v)$ option.

COMMIX 1AR/P consists of approximately 150 subroutines. Table 3.15 shows that for those data sets examined, the nine listed subroutines consume over $60 \%$ of the time in all cases. Comparing with the baseline runs presented in Tables 3.3-3.5, we notice that the percentage of time spent. in SOLVEV is almost halved by the additional vectorization that is achieved after preprocessing with the dependency analyzer FPP. This gives an indication of the advantages one can sometimes achieve when using more advanced automatic vectorization techniques.

Comparing Table 3.15 with Tables 3.3-3.5, we note that the small differences in the number of calls shown for some subroutines is due to the different methods of accounting used by PERFTRACE and FLOWTRACE.

Table 3.16 shows how the best MFLOPS rate is achieved for the solvEv subroutine. With the exception of LOWFCV - whose relative weight in the total runtime of the code is much smaller than SOLVEV's - the achieved rate is far superior than all other listed subroutines.

Regarding LOWFCV, we note that its superior performance for the simple SV compilation option, is due to the use of the IVDEP inline Cray compiler directives CDIR\$. 
Table 3.3: Characteristics of most time-consuming subroutines of COMMIX-1AR/P for data set P1r0 running in baseline SV mode on Cray X-MP (from PERFTRACE).

\begin{tabular}{||c|l|r|r|r||}
\hline Rank & Program Unit & Times Called & \% Execute & MFLOPS \\
\hline 1 & SOLVEV & 1000 & 14.76 & 10.29 \\
2 & QSTRDS & 1001 & 11.52 & 6.20 \\
3 & ENERGI & 1000 & 10.97 & 6.06 \\
4 & ZMOMI & 1000 & 9.43 & 6.42 \\
5 & YMOMI & 1000 & 6.18 & 6.97 \\
6 & QSTRUC & 1001 & 4.68 & 6.67 \\
7 & PEQN & 1000 & 4.53 & 3.05 \\
8 & XMOMI & 1000 & 4.31 & 6.93 \\
9 & LOWFCV & 26303 & 3.78 & 30.98 \\
10 & HSTRUC & 1001 & 3.44 & 2.13 \\
\hline
\end{tabular}

Table 3.4: Characteristics of most time-consuming subroutines of COMMIX-1AR/P for data set. P1r1 running in baseline SV mode on Cray X-MP (from PERfTrace).

\begin{tabular}{||c|l|r|r|r||}
\hline Rank & Program Unit & Times Called & \% Execute & MFLOPS \\
\hline 1 & SOLVEV & 100 & 18.31 & 10.26 \\
2 & QSTRDS & 100 & 11.48 & 6.20 \\
3 & ENERGI & 100 & 10.94 & 6.06 \\
4 & ZMOMI & 100 & 9.40 & 6.42 \\
5 & YMOMI & 100 & 6.16 & 6.97 \\
6 & QSTRUC & 100 & 4.66 & 0.67 \\
7 & PEQN & 100 & 4.52 & 3.05 \\
8 & XMOMI & 100 & 4.30 & 6.93 \\
\hline
\end{tabular}

Table 3.5: Characteristics of most time-consuming subroutines for data set P1r2 running in baseline SV mode on Cray X-MP (from PERfTRACE).

\begin{tabular}{||c|l|r|r|r||}
\hline Rank & Program Unit & Times Called & \% Execute & MFLOPS \\
\hline 1 & QSTRDS & 12998 & 15.18 & 6.19 \\
2 & ENERGI & 12998 & 14.45 & 6.06 \\
3 & ZMOMI & 12998 & 12.41 & 6.42 \\
4 & YMOMI & 12998 & 8.13 & 6.97 \\
5 & QSTRUC & 12998 & 6.17 & 6.66 \\
6 & PEQN & 12998 & 5.96 & 3.06 \\
7 & XMOMI & 12998 & 5.67 & 6.93 \\
8 & HSTRUC & 12998 & 4.53 & 2.15 \\
\hline
\end{tabular}


Table 3.6: Characteristics of most time-consuming subroutines for data set $\mathrm{P} 2$ running in baseline SV mode on Cray X-MP (from PERFTRACE).

\begin{tabular}{||c|l|r|r|r||}
\hline Rank & Program Unit & Times Called & \% Execute & MFLOPS \\
\hline 1 & QSTR'DS & 65 & 10.32 & 7.71 \\
2 & ENERGI & 64 & 8.27 & 2.84 \\
3 & HSTRUC & 65 & 7.17 & 1.12 \\
4 & ZMOMI & 64 & 7.15 & 5.33 \\
5 & DUCTWA & 130 & 6.97 & 1.70 \\
6 & TLIQ & 51321 & 6.85 & 1.73 \\
7 & QSTRUC & 65 & 5.31 & 7.46 \\
8 & LOWFCV & 3961 & 5.02 & 22.53 \\
\hline
\end{tabular}

Table 3.7: Characteristics of most time-consuming subroutines for data set P3 running in baseline SV mode on Cray X-MP (from PERFTRACE).

\begin{tabular}{||c|l|r|r|r||}
\hline Rank & Program Unit & Times Called & \% Execute & MFLOPS \\
\hline 1 & ZMOMI & 459 & 16.12 & 6.69 \\
2 & ENERGI & 459 & 15.18 & 3.92 \\
3 & XMOMI & 459 & 12.37 & 7.31 \\
4 & YMOMI & 459 & 10.56 & 7.25 \\
5 & PEQN & 459 & 7.38 & 3.25 \\
6 & SOLVEV & 459 & 4.94 & 35.87 \\
7 & SORTC & 459 & 4.60 & 0.00 \\
8 & LOWFCV & 9090 & 4.15 & 30.15 \\
\hline
\end{tabular}

Table 3.8: HPM group 0 summary for baseline SV COMMIX-1AR/P code running on Cray XMP/14 with data sets P1, P2 and P3.

\begin{tabular}{|c|c|c|c|c|c|c|}
\hline & P1r0 & P1rl & P1r2 & P1r3 & P2 & P3 \\
\hline Million inst $/ \sec$ (MIPS) & 37.23 & 37.91 & 36.45 & 36.62 & 35.70 & 35.98 \\
\hline Avg. clock periods/inst & 3.16 & 3.10 & 3.23 & 3.21 & 3.30 & 3.27 \\
\hline \% CP holding issue & 49.16 & 48.0 & 48.75 & 48.31 & 50.67 & 49.45 \\
\hline Inst.buffer fetches/sec & $0.40 \mathrm{M}$ & $0.40 \mathrm{M}$ & $0.47 \mathrm{M}$ & $0.47 \mathrm{M}$ & $0.47 \mathrm{M}$ & $0.47 \mathrm{M}$ \\
\hline Floating adds/sec & $3.77 \mathrm{M}$ & 3.14 & $3.20 \mathrm{M}$ & $2.94 \mathrm{M}$ & $4.46 \mathrm{M}$ & $4.04 \mathrm{M}$ \\
\hline Floating multiplies/sec & $4.84 \mathrm{M}$ & $4.28 \mathrm{M}$ & $4.02 \mathrm{M}$ & 3.74 & $6.31 \mathrm{M}$ & $5.45 \mathrm{M}$ \\
\hline Floating reciprocal/sec & 0.51 & 0.51 & 0.46 & $0.45 \mathrm{M}$ & $0.65 \mathrm{M}$ & $0.53 \mathrm{M}$ \\
\hline I/O mem. references/sec & $0.57 \mathrm{M}$ & $0.55 \mathrm{M}$ & $0.55 \mathrm{M}$ & $0.70 \mathrm{M}$ & $0.11 \mathrm{M}$ & $0.37 \mathrm{M}$ \\
\hline CPU mem. references $/ \mathrm{sec}$ & $16.32 \mathrm{M}$ & $13.93 \mathrm{M}$ & $13.30 \mathrm{M}$ & $12.15 \mathrm{M}$ & $18.70 \mathrm{M}$ & $19.04 \mathrm{M}$ \\
\hline Floating ops/CPU second & $9.12 \mathrm{M}$ & $7.92 \mathrm{M}$ & $7.68 \mathrm{M}$ & $7.14 \mathrm{M}$ & $11.43 \mathrm{M}$ & 10.02 \\
\hline
\end{tabular}


Table 3.9: HPM group 1 summary for baseline SV COMMIX-1AR/P code running on Cray $X$ $\mathrm{MP} / 14$ with data sets P1, P2 and P3.

\begin{tabular}{||l|r|r|r|r||r|r||}
\hline & \multicolumn{7}{|c|}{ \% of all CPs } \\
& P1r0 & P1r1 & P1r2 & P1r3 & P2 & P3 \\
\hline Waiting on A-registers/funct. units & 9.54 & 0.61 & 10.16 & 10.25 & 9.42 & 10.29 \\
Waiting on S-registers/funct. units & 27.91 & 28.35 & 3.40 & 31.15 & 28.74 & 26.55 \\
Waiting on V-registers & 5.39 & 4.65 & 2.71 & 2.81 & 0.25 & $\vdots$ \\
Waiting on vector functional units & 3.63 & 3.24 & 0.13 & 2.45 & 3.69 & 3.81 \\
Waiting on scalar memory references & 0.10 & 0.10 & 0.13 & 0.13 & 0.19 & 0.16 \\
Waiting on block mernory references & 4.35 & 3.70 & 2.22 & 1.77 & 4.82 & 5.04 \\
\hline
\end{tabular}

Table 3.10: HPM group 2 summary for baseline SV COMMIX-1AR/P code running on Cray $X$ $\mathrm{MP} / 14$ with data sets $\mathrm{P} 1, \mathrm{P} 2$ and $\mathrm{P} 3$.

\begin{tabular}{|c|c|c|c|c|c|c|}
\hline & \multicolumn{6}{|c|}{$\%$ of all CPs } \\
\hline & Plro & P1r1 & P1r2 & P1r3 & P2 & P3 \\
\hline Inst, buffer fetches/sec & $0.40 \mathrm{M}$ & $0.40 \mathrm{M}$ & $0.47 \mathrm{M}$ & $0.47 \mathrm{M}$ & $0.47 \mathrm{M}$ & $0.47 \mathrm{M}$ \\
\hline Scalar memory refs/sec & $5.79 \mathrm{M}$ & $5.81 \mathrm{M}$ & $6.82 \mathrm{M}$ & $6.95 \mathrm{M}$ & $5.68 \mathrm{M}$ & $7.08 \mathrm{M}$ \\
\hline$\%$ having conflicts & 28.38 & 28.55 & 30.61 & 30.53 & 42.50 & 41.06 \\
\hline I/O memory refs/sec & $0.48 \mathrm{M}$ & $0.69 \mathrm{M}$ & $0.64 \mathrm{M}$ & $0.57 \mathrm{M}$ & $0.16 \mathrm{M}$ & $0.33 \mathrm{M}$ \\
\hline$\%$ having conflicts & 56.74 & 49.84 & 51.37 & 48.76 & 71.50 & 65.61 \\
\hline Block memory refs/sec & $10.53 \mathrm{M}$ & $8.12 \mathrm{M}$ & $6.47 \mathrm{M}$ & $5.21 \mathrm{M}$ & $13.02 \mathrm{M}$ & $11.96 \mathrm{M}$ \\
\hline$\%$ he & 62.43 & 68.61 & 75.91 & 84.24 & & 73.37 \\
\hline $\mathrm{refs} / \mathrm{sec}$ & $10.19 \mathrm{M}$ & $7.79 \mathrm{M}$ & $6.15 \mathrm{M}$ & $4.90 \mathrm{M}$ & $11.71 \mathrm{M}$ & $11.70 \mathrm{M}$ \\
\hline
\end{tabular}

Table 3.11: HPM group 3 summary for baseline SV COMMIX-1AR/P code running on Cray $X$ $\mathrm{MP} / 14$ with data set P1.

\begin{tabular}{|c|c|c|c|c|c|c|c|c|}
\hline \multirow[b]{2}{*}{ type of instruction } & \multicolumn{2}{|c|}{$\mathrm{P} 1 \mathrm{r}()$} & \multicolumn{2}{|c|}{ P1r1 } & \multicolumn{2}{|c|}{ P1r2 } & \multicolumn{2}{|c|}{ P1r3 } \\
\hline & $\begin{array}{l}\text { inst. per } \\
\text { CPU sec }\end{array}$ & $\begin{array}{l}\% \text { of all } \\
\text { inst. }\end{array}$ & $\begin{array}{l}\text { inst, per } \\
\text { CPU sec }\end{array}$ & $\begin{array}{l}\% \text { of all } \\
\text { inst. }\end{array}$ & $\begin{array}{l}\text { inst. per } \\
\text { CPU sec }\end{array}$ & $\begin{array}{c}\% \text { of all } \\
\text { inst. }\end{array}$ & $\begin{array}{l}\text { inst. per } \\
\text { CPU sec }\end{array}$ & $\begin{array}{l}\% \text { of all } \\
\text { inst. }\end{array}$ \\
\hline jump/special & $3.47 \mathrm{M}$ & 9.32 & $3.64 \mathrm{M}$ & 9.59 & $3.15 \mathrm{M}$ & 8.64 & $3.18 \mathrm{M}$ & 8.67 \\
\hline scalar & $32.94 \mathrm{M}$ & 88.50 & $33.59 \mathrm{M}$ & 88.57 & $32.67 \mathrm{M}$ & 89.64 & $32.89 \mathrm{M}$ & 89.80 \\
\hline vector integer/logical & $0.19 \mathrm{M}$ & 0.51 & $0.21 \mathrm{M}$ & 0.55 & $0.20 \mathrm{M}$ & 0.55 & 0.20 & 0,55 \\
\hline vector floating point & $0.20 \mathrm{M}$ & 0.55 & $0.15 \mathrm{M}$ & 0.4 & $0.13 \mathrm{M}$ & 0.36 & $0.11 \mathrm{M}$ & 0.29 \\
\hline vector memory & $0.42 \mathrm{M}$ & 1.12 & $0.33 \mathrm{M}$ & 0.8 & $0.29 \mathrm{M}$ & 0.81 & $0.25 \mathrm{M}$ & 0,09 \\
\hline type of operation & $\begin{array}{l}\text { ops per } \\
\text { CPUsec }\end{array}$ & $\begin{array}{r}\text { avg. } \\
\text { VL }\end{array}$ & $\begin{array}{l}\text { ops per } \\
\text { CPU Usec }\end{array}$ & $\begin{array}{l}\text { avg. } \\
\text { VL }\end{array}$ & $\begin{array}{l}\text { ops per } \\
\text { CPUsec }\end{array}$ & $\begin{array}{l}\text { avg. } \\
\text { VL }\end{array}$ & $\begin{array}{l}\text { ops per } \\
\text { CPUsec }\end{array}$ & $\begin{array}{l}\text { avg. } \\
\text { VI. }\end{array}$ \\
\hline Vector integer\&logical & $3.22 \mathrm{M}$ & 16.91 & $4.23 \mathrm{M}$ & 20.30 & $2.12 \mathrm{M}$ & 10.61 & $2.04 \mathrm{M}$ & 10.1 .1 \\
\hline Vector floating point & $5.11 \mathrm{M}$ & 24.99 & $3.92 \mathrm{M}$ & 25.57 & $2.94 \mathrm{M}$ & 22.32 & $2.31 \mathrm{M}$ & 21.53 \\
\hline Vector memory & $10.19 \mathrm{M}$ & 24.41 & $7.79 \mathrm{M}$ & 23.41 & $6.15 \mathrm{M}$ & 20.87 & $4.90 \mathrm{M}$ & 19.35 \\
\hline
\end{tabular}


Table 3.12: HPM group 3 summary for baseline SV COMMIX-1AR/P code running on Cray $X$ $\mathrm{MP} / 14$ with data sets $\mathrm{P} 2$ and $\mathrm{P} 3$.

\begin{tabular}{|c|c|c|c|c|}
\hline \multirow[b]{2}{*}{ type of instruction } & \multicolumn{2}{|c|}{ P2 } & \multicolumn{2}{|c|}{$\overline{\mathrm{P} 3}$} \\
\hline & $\begin{array}{l}\text { inst. per } \\
\text { CPU sec. }\end{array}$ & $\begin{array}{c}\% \text { of all } \\
\text { inst. }\end{array}$ & $\begin{array}{l}\text { inst. per } \\
\text { CPU sec }\end{array}$ & $\begin{array}{l}\% \text { of all } \\
\text { inst. }\end{array}$ \\
\hline jump/special & $3.25 \mathrm{M}$ & 9.10 & $2.57 \mathrm{M}$ & 7.13 \\
\hline scalar & $31.54 \mathrm{M}$ & 88.37 & $32.60 \mathrm{M}$ & 90.57 \\
\hline vector integer/logical & $0.16 \mathrm{M}$ & 0.46 & $0.21 \mathrm{M}$ & 0.58 \\
\hline vector floating point & $0.25 \mathrm{M}$ & 0.70 & $0.20 \mathrm{M}$ & 0.56 \\
\hline vector memory & $0.49 \mathrm{M}$ & 1.36 & $0.42 \mathrm{M}$ & 1.17 \\
\hline type of operation & ops per & avg: & s per & avg. \\
\hline & PUsec & VL & CPUsec & $V L$ \\
\hline Vecto & $3.73 \mathrm{M}$ & 22.62 & $3.65 \mathrm{M}$ & 17.62 \\
\hline Vecto & $5.71 \mathrm{M}$ & 22.93 & $5.88 \mathrm{M}$ & 29.26 \\
\hline Vector memory & $11.70 \mathrm{M}$ & 24.03 & $11.70 \mathrm{M}$ & 27.88 \\
\hline
\end{tabular}

Table 3.13: Execution times and MFLOPS for SV(Zv) COMMIX-1AR/P code running on Cray $\mathrm{X}-\mathrm{MP} / 48$ (from HPM).

\begin{tabular}{||l|c|c|c|c||}
\hline & P1r0 & P1r1 & P1r2 & P1r3 \\
\hline Time & 82.35 & 8.17 & 880.64 & 628.17 \\
MFLOPS & 11.76 & 10.96 & 8.76 & \\
\hline
\end{tabular}

Table 3.14: Timing profile for COMMIX-1AR/P running on Cray X-MP/48, compiled in SV(Zv) mode.

\begin{tabular}{||l|c|c|c|c||}
\hline Data: & P1r0 & P1r1 & P1r2 & P1r3 \\
\hline Total Execution Time & 77.71 & 7.64 & 869.04 & 622.37 \\
Momentum Eqns. Construction & 21.93 & 2.12 & 297.70 & 204.49 \\
Momentum Eqns. Solution & 20.39 & 1.72 & 220.46 & 149.68 \\
Energy Eqns. Construction & 25.58 & 2.56 & 338.06 & 247.22 \\
Energy Eqns. Solution & 6.48 & .836 & 18.12 & 13.13 \\
Thermal Struct & 2.81 & .281 & 3.10 & 1.13 \\
Thermal Struct Radiation & 0.065 & .0065 & .072 & 0.026 \\
\hline
\end{tabular}


Table 3.15: Profile of the most time consuming subroutines in SV(Zv) mode for COMMIX-1Ali/P running on Cray X-MP/48 (from FLOWTRACE).

\begin{tabular}{||l|c|c|c|c|c|c|c|c||}
\hline Routine & \multicolumn{3}{|c}{ P1r0 } & \multicolumn{2}{c|}{ P1r1 } & \multicolumn{2}{c|}{ P1r'2 } & \multicolumn{2}{c||}{ 1r3 } \\
& Calls & (percent) & Calls & (percent) & Calls & (percent) & Calls & (percent) \\
\hline QSTRDS & 1001 & 11.49 & 100 & 11.53 & 13156 & 13.99 & 9629 & 14.32 \\
ZMOM1 & 1000 & 9.69 & 100 & 9.69 & 13156 & 11.77 & 9629 & 12.05 \\
ENERG1 & 1000 & 9.52 & 100 & 9.64 & 13156 & 11.73 & 9629 & 12.00 \\
SOLVEV & 1000 & 7.45 & 100 & 9.95 & 13156 & 1.69 & 9629 & 1.72 \\
YMOMI & 1000 & 6.52 & 100 & 6.59 & 13156 & 8.0 & 9629 & 8.19 \\
QSTRUC & 1001 & 5.10 & 100 & 5.12 & 13156 & 6.22 & 9629 & 6.26 \\
PEQN & 1000 & 4.69 & 100 & 4.72 & 13156 & 5.73 & 9629 & 5.87 \\
XMOMI & 1000 & 4.55 & 100 & 4.59 & 13156 & 5.58 & 9629 & 5.71 \\
LOWFCV & 26373 & 4.28 & 1238 & 2.10 & 178939 & 2.86 & 89659 & 2.01 \\
\hline
\end{tabular}

The linear system solver sOLVEv, although expensive in number of computations, has boon written to take advantage of vectorization and thus performs at 35 MFLOPS on one CPU of the Cray X-MP. This is in contrast to a meager average of 6.5 MFLOPS for those sections of the code working on the task of matrix construction. This of course causes the overall performance to drop) to almost 11 MFLOPS, even for the full vector optimization. It is thus clear that a first step in improving the performance of the code is going to be the restructuring of the matrix construction phase.

We remark here that Table 3.16 reveals a great deal about the nature of work to be done during this project:

- The difference in performance for sOLVEV under the different compilation options tells one about the ability of restructuring compilers to take advantage of the machine capabilities, if the code is written properly. It also shows that the use of more sophisticated restructurers can be very beneficial. We should keep in mind however that sOLVEV was coded for vector processing.

- The high performance of LOWFCV for both SV and SV $(Z v)$ shows that even a less sophisticated restructurer can do well if it has some help from the user (in the form of inline directives).

- The low performance for both SV and SV(Zv) options for all other routines, shows that there is work to be done until these cornmercial compilers can handle satisfactorily dusty-decks ("dusty-decks" as the matrix assembly routines were not coded to take advantage of the architecture.) We will thus investigate the use of novel restructurer's as part of Phase 2 of our work.

- The overall low performance for buth compilation options, and the small difference amongst, the two, shows that it is dangerous to concentrate only on those stages of the code which are related to well defined algebraic computations in need of new algorithms (e.g. linear system solvers), at least until the automatic transformation tools become more powerful.

Finally, Tables 3.1 and 3.17 show the performance of the code when run in the multiprocessing environments of the Alliant $\mathrm{FX} / 80$ and the Cray $\mathrm{X}-\mathrm{MP} / 48$, with restructuring performed auto- 
Table 3.16: MFLOPS profile of the most time consuming subroutines in baseline SV, and enhanced vectorization $\mathrm{SV}(\mathrm{Zv})$ modes for the COMMIX-1AR/P code running on Cray X-MP using data decks P1r0 and P1r2 (from PERF'TRACE).

\begin{tabular}{||l|c|c||c|c||}
\hline Data set & \multicolumn{2}{|c|}{ P1r0 } & \multicolumn{2}{c||}{ P1r2 } \\
\hline Routine & SV & SV(Zv) & SV & SV(Zv) \\
\hline QSTRDS & 6.20 & 6.68 & 6.19 & 6.69 \\
ZMOMI & 6.42 & 7.19 & 6.42 & 7.15 \\
ENERGI & 6.06 & 6.61 & 6.06 & 6.64 \\
SOLVEV & 10.29 & 36.28 & 10.37 & 33.51 \\
YMOMI & 6.97 & 7.78 & 6.97 & 7.81 \\
QSTRUC & 6.67 & 7.28 & 6.66 & 7.29 \\
PEQN & 3.05 & 3.49 & 3.06 & 3.50 \\
XMOM1 & 6.93 & 7.82 & 6.93 & 7.84 \\
LOWFCV & 30.98 & 34.12 & 30.99 & 34.57 \\
\hline Overall & 9.12 & 11.76 & 7.68 & 8.76 \\
\hline
\end{tabular}

Table 3.17: Execution time profile for the SVC version of COMMIX-1AR/P running on Cray $\mathrm{X}-\mathrm{MP} / 48$ (from HPM).

\begin{tabular}{||l|c|c|c|c||}
\hline Concurrency & P1r0 & P1r2 & Connect seconds & Connect $\times$ CPUs \\
\hline 1 CPU & 22.25 & 297.28 & 412.6 & 412.6 \\
2 CPU & 31.76 & 405.18 & 303.3 & 606.5 \\
3 CPU & 47.19 & 461.88 & 231.9 & 695.9 \\
4 CPU & 123.95 & 861.20 & 364.5 & 1458.1 \\
\hline Totals & 225.25 & 2025.48 & 1312.4 & 3173.2 \\
\hline
\end{tabular}

matically by the respective compilers. It is clear that multiprocessing is of little benefit without manual intervention.

Table 3.17 gives the job accounting information for the vector-concurrent version. This information provides the connect time in each active CPU and the number of active CPUs, Let $T_{i}$, $i=1, \ldots, 4$, be the connect time in each active CPU when $i$ CPUs are active. The total execution time and total CPU time, as shown in the last two rows of this table, are defined as :

$$
\begin{aligned}
\text { total execution time } & =T_{1}+T_{2}+T_{3}+T_{4}, \text { and } \\
\text { total CPU time } & =T_{1}+2 T_{2}+3 T_{3}+4 T_{4} .
\end{aligned}
$$

The average number of concurrent CPUs is thus the ratio of the lotal number of connect-seconds and the ConnectxCPUs product. Thus $3173.2720 / 1312.4346=2.42$.

The following observations can be drawn regarding the statistics reported in Table 3.18.

1. For the performance to be considered acceptable, the MVLOPS (last row in Table 3.18) should be between 20 and $200 \mathrm{MFLOPS}$; the average MFLOPS for vector SV(Zv) code is about 9 
Table 3.18: HPM group 0 summary for SV(Zv) and SVC COMMIX-1AR/P code running on Cray $\mathrm{X}-\mathrm{MP} / 48$ with data set $\mathrm{P} 1$.

\begin{tabular}{|c|c|c|c|c|c|c|c|c|}
\hline \multirow[t]{2}{*}{ Category } & \multicolumn{4}{|c|}{$\mathrm{SV}(\mathrm{ZV})$} & \multicolumn{4}{|c|}{ SVC } \\
\hline & P1r0 & P1rl & P1r2 & P1r3 & Plro & P1rl & P1r2 & Ple3 \\
\hline Million inst/sec (MIPS) & 37.11 & 30.92 & 38,62 & & 16.70 & 19.03 & 17.83 & 16.31 \\
\hline Avg. clock periods/inst & 3.17 & 3.10 & 3.05 & & 7.01 & 6.18 & 6,60 & 7.22 \\
\hline \% CP holding issue & 52.61 & 52.49 & 50.09 & & 78.88 & 76.25 & 77.11 & 79.07 \\
\hline Inst, buffer letches $/ \mathrm{sec}$ & $0,44 \mathrm{M}$ & $0.45 \mathrm{M}$ & $0.47 \mathrm{M}$ & & $0.17 \mathrm{M}$ & $0.19 \mathrm{M}$ & $0.21 \mathrm{M}$ & $0.20 \mathrm{M}$ \\
\hline Floating adds/sec & $4.76 \mathrm{M}$ & $4.17 \mathrm{M}$ & $3.84 \mathrm{M}$ & & $1.71 \mathrm{M}$ & $1.63 \mathrm{M}$ & $1.66 \mathrm{~N}^{\prime}$ & $1.41 \mathrm{M}$ \\
\hline Floating multiplies/sec & $6.34 \mathrm{M}$ & $6.08 \mathrm{M}$ & $4.41 \mathrm{M}$ & & $2.03 \mathrm{M}$ & $2.02 \mathrm{M}$ & $1.85 \mathrm{M}$ & $1.57 \mathrm{M}$ \\
\hline Floating reciprocal/sec & $0.67 \mathrm{M}$ & $0.70 \mathrm{M}$ & $0.50 \mathrm{M}$ & & $0.21 \mathrm{M}$ & $0.24 \mathrm{M}$ & $0.21 \mathrm{M}$ & $0.19 \mathrm{M}$ \\
\hline $1 / 0 \mathrm{mem}$. references/sec & $0.18 \mathrm{M}$ & $0.33 \mathrm{M}$ & $0.49 \mathrm{M}$ & & $0.14 \mathrm{M}$ & $0.22 \mathrm{M}$ & $0.23 \mathrm{M}$ & $0.11 \mathrm{M}$ \\
\hline CPU mern, references/sec & $21.12 \mathrm{M}$ & $19.14 \mathrm{M}$ & $16.25 \mathrm{M}$ & & $7.62 \mathrm{M}$ & $7.49 \mathrm{M}$ & $7.09 \mathrm{M}$ & $6.98 \mathrm{M}$ \\
\hline Floating ops/CPU second & $11.76 \mathrm{M}$ & $10.96 \mathrm{M}$ & $8.76 \mathrm{M}$ & & $3.96 \mathrm{M}$ & $3.88 \mathrm{M}$ & $3.73 \mathrm{M}$ & $3.17 \mathrm{M}$ \\
\hline
\end{tabular}

Table 3.19: Ratio of scalar and vector floating operations for COMMIX-1AR/P code running on Cray X-MP/48 with data set $\mathrm{P} 1$.

\begin{tabular}{|c|c|c|c|c||r|r|r|r||}
\hline & \multicolumn{4}{|c|}{ SV(Zv) } & \multicolumn{5}{|c|}{ SVC } \\
\hline Ratlo & 1.62 & 1.42 & 0.74 & & 2.85 & 1.11 & 0.79 & 0.71 \\
\hline
\end{tabular}

MFLOPS which is very low. The reported MFLOPS for vector-concurrent (-Zp) code (SVC In our notation) is even lower, an average 3.6. But one has to keep in mind that the code was running on up to four CPUs in multiuser mode. An average of $2.42 \mathrm{CP}$ Us was used for the runs that produced the statistics in the table. The average MFLOPS is thus $8.9(3.6 \times 2.42)$ for the SVC version of the code.

2. The MIPS rate for the Cray X-MP should be between 20 and 80 . For vector code the average MIPS is about 37. A low value accompanied with high MFLOPS indicates long vector instructions. This is not the case here indicating that code should be vectorized further.

3. The MFLOPS rate reported at the bottom of the table includes scalar and vector floating point operations. An important measure of extent of vectorization is the vector to scalar floating operations ratio. The HPM group 3 statistics report the vector floating point operations. Such data reported later in Tables 3.22 and 3.23 , can be used in conjunction with Table 3.18 to compute the vector/scalar ratio. Such ratios were computed and are presented in Table 3.19. These ratios are very low indicating that the code is spending most of its time in scalar operations.

The following observations can be drawn regarding the statistics reported in Table 3.20.

1. For multitasked (concurrent) code, a very large (55) percentage of the execution lime was spent waiting (to synchronize) on semaphores.

2. The figure for "waiting on S-registers/funct, units", i.e., scalar registers and functional units, is also high compared to "waiting on V-registers" and "wajting on vector functional units". This shows that the code is spending more of its time in scalar mode. 
Table 3.20: HPM group 1 summary for SV(Zv) and SVC COMMIX-1AR/P code running on (ray $\mathrm{X} \cdot \mathrm{MP} / 48$ with data set $\mathrm{P} 1$.

\begin{tabular}{||l|r|r|r|r|r|r|r|r||}
\hline Category & \multicolumn{3}{|c||}{ SV(Zv) } & \multicolumn{4}{c||}{ \%VC } \\
& P1r0 & P1r1 & P1r2 & P1r3 & P1r0 & P1r1 & P1r2 & P1r3 \\
\hline Waiting on semaphores & 0.00 & 0.00 & 0.00 & 0.00 & 55.63 & 51.61 & 58.98 & 62.47 \\
Walting on shared registers & 0.00 & 0.00 & 0.00 & 0.00 & 0.18 & 0.30 & 0.15 & 0.15 \\
Waiting on A-registers/funct. units & 9.11 & 9.07 & 9.76 & 9.83 & 3.93 & 4.14 & 4.02 & 3.69 \\
Waiting on S-registers/funct. units & 26.46 & 26.69 & 29.13 & 29.62 & 11.37 & 12.49 & 11.89 & 11.05 \\
Waiting on V-registers & 6.75 & 6.62 & 3.87 & 3.32 & 2.32 & 2.30 & 1.44 & 1.13 \\
Waiting on vector functional units & 6.10 & 6.56 & 3.82 & 3.65 & 1.73 & 1.79 & 1.37 & 1.19 \\
Wailting on scalar memory references & 0.89 & 0.99 & 1.12 & 0.81 & 0.16 & 0.21 & 0.16 & 0.13 \\
Waiting on block memory references & 6.40 & 5.84 & 4.10 & 3.43 & 2.31 & 2.21 & 1.47 & 1.17 \\
\hline
\end{tabular}

The following observations can be drawn regarding the statistics reported in Table 3.21 .

1. A figure for "inst. buffer fetches/sec" larger than 1 million per second indicates "spaghetti" code. The numbers reported by group 2 statistics show this is not the case.

2. The comparison of scalar to vector memory references provides a measure for the extent of vectorization. The statistics show a ratio of vector to scialar references ranging from 0.93 to 2.23. This range is low:

3. A high "block memory ref./sec" rate with low scalar and vector memory reference rate would indicate that the code is spending too much time in entry and exit code of the subroutines and functions. This could happen if subroutines were called but did very little work. This is not the case. The block memory reference rate is comparable to vector and scalar reference rate.

The following observations can be drawn regarding the statistics reported in Tables 3.22 and 3.23 .

1. The number of scalar instructions is overwhelmingly high, almost 88 percent of all instructions. But this can be misleading because it includes non-floating point instructions also. The ratio of the vector to scalar floating point operations reported in Table 3.19 is more indicative of the level of vectorization.

2. The average length per vector floating-point instruction, as reported in Table 3.22 , is about 23, much lower than the length of the vector registers (64) and even lower than 53 and 45 , the published measurements of $n_{1 / 2}$ for dyadic and triadic operations respectively, the vector length required to achieve half the maximum asymptotic performance (in this case taken to be 70 and 103 MFLOPS) [8].

\subsubsection{Alliant FX/80}

The following changes were needed to port the $1 \mathrm{AR} / \mathrm{P}$ code. 
Table 3.21: HPM group 2 summary for SV(Zv) and SVC COMMXX 1 AR/P code running on 4-CIU Cray X-MP/48 with data set P1.

\begin{tabular}{||l|r|r|r|r|r|r|r|r||}
\hline Category & \multicolumn{9}{|c|}{ SC(Zv) } & \multicolumn{3}{|c||}{ SVC } \\
& $\mathrm{P} 1 \mathrm{r} 0$ & $\mathrm{P} 1 \mathrm{r} 1$ & $\mathrm{P} 1 \mathrm{r} 2$ & $\mathrm{P} 1 \mathrm{r} 3$ & $\mathrm{P} 1 \mathrm{r} 0$ & $\mathrm{P} 1 \mathrm{r} 1$ & $\mathrm{P} 1 \mathrm{r} 2$ & $\mathrm{P} 1 \mathrm{r} 3$ \\
\hline Inst. buffer fetches/sec & $0.45 \mathrm{M}$ & $0.46 \mathrm{M}$ & $0.48 \mathrm{M}$ & $0.48 \mathrm{M}$ & $0.17 \mathrm{M}$ & $0.17 \mathrm{M}$ & $0.20 \mathrm{M}$ & $0.22 \mathrm{M}$ \\
Scalar memory refs/sec & $6.50 \mathrm{M}$ & $6.57 \mathrm{M}$ & $7.39 \mathrm{M}$ & $7.52 \mathrm{M}$ & $2.56 \mathrm{M}$ & $2.67 \mathrm{M}$ & $3.05 \mathrm{M}$ & $3.34 \mathrm{M}$ \\
\% having conflicts & 47.75 & 44,69 & 44.98 & 43.72 & 22.6 & 23.9 & 27.7 & 28.5 \\
I/O memory refs/sec & $0.18 \mathrm{M}$ & $0.09 \mathrm{M}$ & $0.11 \mathrm{M}$ & $0.30 \mathrm{M}$ & $0.07 \mathrm{M}$ & $0.18 \mathrm{M}$ & $0.16 \mathrm{M}$ & $0.07 \mathrm{M}$ \\
\% having conflicts & 43.49 & 50.83 & 40.38 & 41.18 & 22.6 & 12.4 & 29.1 & 32.2 \\
Block memory refs/sec & $14.90 \mathrm{M}$ & $12.91 \mathrm{M}$ & $9.11 \mathrm{M}$ & $7.85 \mathrm{M}$ & $5.00 \mathrm{M}$ & $4.20 \mathrm{M}$ & $3.58 \mathrm{M}$ & $3.29 \mathrm{M}$ \\
\% having conflicts & 33.30 & 32.63 & 25.42 & 24.43 & 20.8 & 21.1 & 19.6 & 18.8 \\
Vector memory refs/sec & $14.48 \mathrm{M}$ & $12.49 \mathrm{M}$ & $8.73 \mathrm{M}$ & $7.49 \mathrm{M}$ & $4.82 \mathrm{M}$ & $4.02 \mathrm{M}$ & $3.42 \mathrm{M}$ & $3.12 \mathrm{M}$ \\
\hline
\end{tabular}

Table 3.22: HPM group 3 summary for $\mathrm{SV}(\mathrm{Zv})$ COMMIX-1AR/P code running on Cray X-MP/48 with data set $P 1$.

\begin{tabular}{|c|c|c|c|c|c|c|c|c|}
\hline \multirow[b]{2}{*}{ type of instruction } & \multicolumn{2}{|c|}{ P1r0 } & \multicolumn{2}{|c|}{ P1r1 } & \multicolumn{2}{|c|}{ P1r2 } & \multicolumn{2}{|c|}{ P1 13} \\
\hline & $\begin{array}{l}\text { inst. per } \\
\text { CPU sec }\end{array}$ & $\begin{array}{l}\% \text { of all } \\
\text { inst. }\end{array}$ & $\begin{array}{l}\text { inst. per } \\
\text { CPU sec }\end{array}$ & $\begin{array}{l}\% \text { of all } \\
\text { inst. }\end{array}$ & $\begin{array}{l}\text { inst. per } \\
\text { CPU sec }\end{array}$ & $\begin{array}{l}\% \text { of all } \\
\text { inst. }\end{array}$ & $\begin{array}{l}\text { inst. per } \\
\text { CPU gec }\end{array}$ & $\begin{array}{c}\% \text { of all } \\
\text { inst. }\end{array}$ \\
\hline jump/special & $3.31 \mathrm{M}$ & 8.77 & $3.34 \mathrm{M}$ & 8.86 & $3.42 \mathrm{M}$ & 8.69 & $3.44 \mathrm{M}$ & 8.71 \\
\hline scalar & $33.22 \mathrm{M}$ & 88.02 & $33.21 \mathrm{M}$ & 88.11 & $34.96 \mathrm{M}$ & 89.00 & $35.16 \mathrm{M}$ & 80.11 \\
\hline vector integer/logical & $0.38 \mathrm{M}$ & 1.01 & $0.43 \mathrm{M}$ & 1.14 & $0.35 \mathrm{M}$ & 0.88 & $0.35 \mathrm{M}$ & 0.89 \\
\hline vector floating point & $0.27 \mathrm{M}$ & 0.73 & $0.23 \mathrm{M}$ & 0.60 & $0.18 \mathrm{M}$ & 0.45 & $0.15 \mathrm{M}$ & 0.38 \\
\hline vector memory & $0.56 \mathrm{M}$ & 1.48 & $0.48 \mathrm{M}$ & 1.29 & $0.39 \mathrm{M}$ & 0.98 & $0.34 \mathrm{M}$ & 0.87 \\
\hline type of operation & $\begin{array}{l}\text { ops per } \\
\text { CPUsec }\end{array}$ & $\begin{array}{l}\text { avg. } \\
\text { VL }\end{array}$ & $\begin{array}{l}\text { ops per } \\
\text { CPUsec }\end{array}$ & $\begin{array}{l}\text { avg. } \\
\text { VL }\end{array}$ & $\begin{array}{l}\text { ops per } \\
\text { CPUsec }\end{array}$ & $\begin{array}{l}\text { avg. } \\
\text { VL }\end{array}$ & $\begin{array}{l}\text { ops per } \\
\text { CPUser }\end{array}$ & $\begin{array}{l}\text { avg. } \\
\text { VI. }\end{array}$ \\
\hline Vector integer\&logical & $8.82 \mathrm{M}$ & 23.20 & $11.15 \mathrm{M}$ & 25.92 & $5.56 \mathrm{M}$ & 16.06 & $5.54 \mathrm{M}$ & 15.82 \\
\hline Vector floating point & $7.27 \mathrm{M}$ & 26.43 & $8.44 \mathrm{M}$ & 28.30 & $3.72 \mathrm{M}$ & 21.11 & $3.08 \mathrm{M}$ & 20.28 \\
\hline Vector memory & $14.54 \mathrm{M}$ & 26.01 & $12.56 \mathrm{M}$ & 25.92 & $8.75 \mathrm{M}$ & 22.70 & $7.50 \mathrm{M}$ & 21.81 \\
\hline
\end{tabular}


Table 3.23: HPM group 3 summary for SVC COMMIX-1AR/P code running on Cray X-MP/48 with data set $\mathrm{P} 1$.

\begin{tabular}{|c|c|c|c|c|c|c|c|c|}
\hline \multirow[b]{2}{*}{ type of instruction } & \multicolumn{2}{|c|}{ P1r0 } & \multicolumn{2}{|c|}{ P1r1 } & \multicolumn{2}{|c|}{ P1r2 } & \multicolumn{2}{|c|}{$\mathrm{P} 1 \mathrm{r} 3$} \\
\hline & $\begin{array}{l}\text { inst. per } \\
\text { CPU sec }\end{array}$ & $\begin{array}{c}\% \text { of all } \\
\text { inst. }\end{array}$ & $\begin{array}{l}\text { inst. per } \\
\text { CPU sec }\end{array}$ & $\begin{array}{r}\% \text { of all } \\
\text { inst. }\end{array}$ & $\begin{array}{l}\text { inst. per } \\
\text { CPU sec }\end{array}$ & $\begin{array}{r}\% \text { of all } \\
\text { inst. }\end{array}$ & $\begin{array}{l}\text { inst. per } \\
\text { CPU sec }\end{array}$ & $\begin{array}{l}\% \text { of all } \\
\text { inst. }\end{array}$ \\
\hline jump/special & $2.21 \mathrm{M}$ & 10.27 & $2.00 \mathrm{M}$ & 10.75 & $1.78 \mathrm{M}$ & 9.17 & $1.77 \bar{M}$ & 9.23 \\
\hline scalar & $18.75 \mathrm{M}$ & 87.29 & $16.20 \mathrm{M}$ & 87.14 & $17.24 \mathrm{M}$ & 88.69 & $16.98 \mathrm{M}$ & 88.79 \\
\hline vector integer/logical & $0.15 \mathrm{M}$ & 0.69 & $0.13 \mathrm{M}$ & 0.72 & $0.16 \mathrm{M}$ & 0.81 & $0.15 \mathrm{M}$ & 0.81 \\
\hline vector floating point & $0.12 \mathrm{M}$ & 0.58 & $0.08 \mathrm{M}$ & 0.44 & $0.08 \mathrm{M}$ & 0.42 & $0.07 \mathrm{M}$ & 0.36 \\
\hline vector memory & $0.25 \mathrm{M}$ & 1.17 & $0.18 \mathrm{M}$ & 0.95 & $0.18 \mathrm{M}$ & 0.92 & $0.16 \mathrm{M}$ & 0.81 \\
\hline type of operation & $\begin{array}{l}\text { ops per } \\
\text { CPUsec }\end{array}$ & $\begin{array}{r}\text { avg. } \\
\text { VL }\end{array}$ & $\begin{array}{l}\text { ops per } \\
\text { CPUsec }\end{array}$ & $\begin{array}{l}\text { avg. } \\
\text { vL }\end{array}$ & $\begin{array}{l}\text { ops per } \\
\text { CPUsec }\end{array}$ & $\begin{array}{l}\text { avg. } \\
\text { VL }\end{array}$ & $\begin{array}{l}\text { ops per } \\
\text { CPUsec }\end{array}$ & $\begin{array}{r}\text { avg. } \\
\text { VL }\end{array}$ \\
\hline Vector integer\&logical & $2.80 \mathrm{M}$ & 18.86 & $2.84 \mathrm{M}$ & $\overline{21.26}$ & $2.35 \mathrm{M}$ & 15.03 & $2.29 \mathrm{M}$ & $\overline{14.78}$ \\
\hline Vector floating point & $2.93 \mathrm{M}$ & 23.67 & $1.96 \mathrm{M}$ & 23.88 & $1.65 \mathrm{M}$ & 20.26 & $1.32 \mathrm{M}$ & 19.25 \\
\hline Vector memory & $6.29 \mathrm{M}$ & 24.99 & $4.27 \mathrm{M}$ & 24.32 & $4.00 \mathrm{M}$ & 22.39 & $3.33 \mathrm{M}$ & 21.43 \\
\hline
\end{tabular}

- Change real data type declarations from single to double precision. For all the compilations we used the $-r 8$ option but there were some explicit conversions necessary to handle explicitly typed double precision reals.

- Change machine dependent constants.

- Substitute calls to sysiem level routines.

- Link with appropriate libraries. In particular we linked with the CSRD mathematical software library to use the functional equivalents of the LINPACK subroutines DGEFA, DGESL. From the CSRD library we used also the functional equivalent of the Cray second timing subroutine, which was written using the etime Alliant intrinsic.

Executable images were produced for each of the SV, SC, and SVC optimization options with the -DAS - $r 8$ options also in effect. The executable image was then run for each of the P1r0, P1r1 and P1r2 data sets.

In the case of COMMIX-1AR/P running on the Alliant FX/80 we were not able to obtain GPROF results whenever concurrency was one of the optimization options, as the code failed upon execution. We are currently investigating this problem. Instead we manually instrumented the code with calls to etime to accumulate the time spent in each of the most important subroutines. In a few cases we also used the system-level time command.

The vector-only code was executed on $1 \mathrm{CE}$.

We first show the summarized timing results for each mode of optimization. This is a yardstick for future performance improvements.

Table 3.24 presents the timing data for the SV, SC, and SVC optimization options. These data were obtained by hand instrumenting the code in its entrance and exit points with etime.

We notice immediately that the speedup obtained is very small, obtaining a maximum of 1.23 (cf. Table 3.25). Given our data from the Cray runs, this is hardly surprising.

An interesting observation which warrants further study is the slight increase of runtime across data sets when choosing an enhanced optimization option, namely SVC instead of SC. This is most 
Table 3.24: Execution times for SV, SC and SVC COMMIX-1AR/P code running on an Alliant $\mathrm{FX} / 80$.

\begin{tabular}{||l|r|r|r||}
\hline Version & P1r0 & P1r1 & P1r2 \\
\hline SV & 1322.4 & 122.3 & 12331.2 \\
SC & 1128.7 & 106.4 & 10015.0 \\
SVC & 1136.7 & 106.6 & 10048.6 \\
\hline
\end{tabular}

Table 3.25: Effect of number of CEs on execution times for SVC COMMIX-1AR/P code running on an Alliant FX/80.

\begin{tabular}{||l|r|r|r|r|r|r||}
\hline & \multicolumn{3}{|c}{ P1r0 } & \multicolumn{2}{c}{ P1r1 } & \multicolumn{2}{c||}{ P1r2 } \\
CEs & Time & Speedup & Time & Speedup & Time & Speedup \\
\hline 1 & 1385.9 & 1.0 & 130.9 & 1.0 & 12393.0 & 1.0 \\
2 & 1230.7 & 1.12 & 114.6 & 1.14 & 11481.7 & 1.07 \\
4 & 1173.5 & 1.18 & 110.7 & 1.18 & 10380.8 & 1.19 \\
6 & 1142.4 & 1.21 & 107.0 & 1.22 & 10462.5 & 1.18 \\
8 & 1136.7 & 1.21 & 106.6 & 1.22 & 10048.6 & 1.23 \\
\hline
\end{tabular}

likely due to the short vector lengths for those loops which VAST transforms to vector-concurrent, form. For a fixed number of CEs, and a loop processed in SVC (vector-concurrent) mode, the overhead involved in issuing and executing a vector instruction per CE will be greater than the time required to issue and execute the few required scalar instructions when operating in SC mode. One can also say that the "efficiency" of the vector instructions is very luw. A witness to the difficulty is the information from HPM group 3 (cf. Table 3.23) for the Cray, which shows the average vector length for $\mathrm{SV}(\mathrm{Zv})$ mode to be only about 23 for each data set. Such a vector length would result in an average iength of $\frac{23}{p}$ for the vector instructions issued when $p$ CEs are executing. For $p=8$, this means that the vector length is only about 3 , which is below the length needed to achieve parity with scalar performance. One option we are currently investigating is the use of the -alt option of the Alliant Fortran compiler which produces alternate versions (scalar or vector) of Do loops, depending on the expected length of the vector statement to be assigned to each processor.

From Table 3.27 we notice that the soLvev routine consumes the maximum percentage of the runtime for P1r0 data. This is in agreement with the data for the SV baseline runs on the Cray (cf. Table 3.3) but in contrast to the SV(Zv) Cray runs presented in Table 3.15, and is due to the inability of the VAST preprocessor to do the necessary concurrent/vector transformations without the special directive corresponding to Cray's IVDEP. As similar remark can be made for LOWFCV.

We note that in Table 3.26 and the ensuing ones, the Time step entry refers to the total time taken by the subroutine TIMSTP. 
Table 3.26: Execution times of various phases for COMMIX-1AR/P code running on an Alliant $\mathrm{FX} / 80$ for P1r0.

\begin{tabular}{||l|r|r|r|r|r|r|r||}
\hline Phase & SV & SC & SVC & SVC & SVC & SVC & SVC \\
& 1ce & 8ce & 1ce & 2ce & 4ce & 6ce & 8ce \\
\hline Time step & 1289.57 & 1101.10 & 1352.57 & 1200.73 & 1145.35 & 1115.55 & 1108.89 \\
Momentum constr. & 296.03 & 271.94 & 291.85 & 281.32 & 290.46 & 284.13 & 283.14 \\
Momentum solu. & 440.76 & 331.55 & 456.68 & 385.51 & 344.50 & 332.94 & 330.08 \\
Energy constr. & 303.03 & 278.38 & 302.38 & 299.97 & 298.95 & 296.31 & 297.49 \\
Energy solu. & 209.51 & 180.28 & 261.52 & 194.75 & 173.75 & 165.31 & 160.88 \\
Thermal Struct. & 19.52 & 19.88 & 19.61 & 19.78 & 19.81 & 19.66 & 19.79 \\
TS Radiation & 1.71 & 1.67 & 1.69 & 1.70 & 1.74 & 1.68 & 1.73 \\
\hline
\end{tabular}

Table 3.27: Execution times of CPU intensive routines in COMMIX-1A.R/P code running on an Alliant FX/80 for P1r0.

\begin{tabular}{||l|r|r|r|r|r|r|r||}
\hline Routine & $\begin{array}{r}\text { S! } \\
\text { 1ce }\end{array}$ & $\begin{array}{r}\text { SC } \\
8 \mathrm{ce}\end{array}$ & $\begin{array}{r}\text { SVC } \\
\text { 1ce }\end{array}$ & $\begin{array}{r}\text { SVC } \\
\text { 2ce }\end{array}$ & $\begin{array}{r}\text { SVC } \\
\text { 4ce }\end{array}$ & $\begin{array}{r}\text { SVC } \\
\text { 6ce }\end{array}$ & $\begin{array}{r}\text { SVC } \\
\text { 8ce }\end{array}$ \\
\hline SOLVEV & 206.58 & 178.10 & 258.40 & 192.30 & 171.63 & 163.28 & 158.91 \\
LOWFCV & & 135.14 & & & & & 136.76 \\
QSTDRS & 76.08 & 70.68 & 82.22 & 76.08 & 76.12 & 77.22 & 79.36 \\
XMOMI & 31.96 & 29.67 & 26.54 & 27.97 & 28.03 & 28.28 & 27.98 \\
YMOMI & 48.93 & 33.74 & 36.22 & 38.15 & 38.52 & 39.80 & 38.39 \\
ZMOMI & 49.08 & 48.97 & 70.18 & 60.74 & 65.95 & 61.31 & 62.61 \\
ENERG1 & 48.94 & 52.04 & 50.20 & 58.29 & 59.04 & 57.17 & 56.54 \\
QSTRUC & 32.21 & 30.01 & 31.83 & 30.70 & 29.54 & 29.08 & 29.12 \\
\hline
\end{tabular}

Table 3.28: Execution times of various phases for COMMIX.1AR/P code running on an Alliant $\mathrm{FX} / 80$ for P1r1.

\begin{tabular}{||l|r|r|r|r|r|r|r||}
\hline Phase & SV & SC & SVC & SVC & SVC & SVC & SVC \\
1ce & 8ce & 1ce & 2ce & 4ce & 6ce & 8ce \\
\hline Time step & 118.26 & 102.93 & 126.18 & 110.30 & 106.53 & 102.91 & 102.49 \\
Momentum constr. & 28.73 & 27.03 & 27.50 & 28.40 & 28.44 & 27.36 & 27.61 \\
Momentum solu. & 29.15 & 21.05 & 30.58 & 24.12 & 22.11 & 21.11 & 20.66 \\
Energy constr. & 29.76 & 27.63 & 30.22 & 30.37 & 29.17 & 29.99 & 30.32 \\
Energy solu. & 26.63 & 23.37 & 33.86 & 23.54 & 23.01 & 20.77 & 20.15 \\
Thermal Struct. & 1.95 & 1.97 & 1.97 & 1.98 & 1.98 & 1.96 & 1.98 \\
TS Radiation & 0.17 & 0.17 & 0.17 & 0.17 & 0.17 & 0.16 & 0.18 \\
\hline
\end{tabular}


Table 3.29: Execution times of CPU intensive routines in COMMIX-1AR/P code running on an. Alliant FX/80 for P1r1

\begin{tabular}{||l|r|r|r|r|r|r|r||}
\hline Routine & $\begin{array}{r}\text { SV } \\
\text { 1ce }\end{array}$ & $\begin{array}{r}\text { SC } \\
\text { 8ce }\end{array}$ & $\begin{array}{r}\text { SVC } \\
\text { 1ce }\end{array}$ & $\begin{array}{r}\text { SVC } \\
\text { 2ce }\end{array}$ & $\begin{array}{r}\text { SVC } \\
\text { 4ce }\end{array}$ & $\begin{array}{r}\text { SVC } \\
\text { 6ce }\end{array}$ & $\begin{array}{r}\text { SVC } \\
8 \mathrm{ce}\end{array}$ \\
\hline SOLVEV & 26.27 & 23.09 & 33.50 & 23.24 & 22.74 & 20.52 & 19.89 \\
LOWFCV & & 6.33 & & & & & 6.13 \\
QSTDRS & 7.29 & 6.70 & 7.86 & 8.61 & 7.35 & 7.55 & 8.12 \\
XMOMI & 3.10 & 3.20 & 2.51 & 2.60 & 2.65 & 2.88 & 2.48 \\
YMOMI & 4.59 & 3.57 & 3.34 & 3.57 & 3.63 & 4.01 & 3.39 \\
ZMOMI & 4.58 & 5.31 & 5.81 & 6.89 & 6.38 & 5.31 & 5.90 \\
ENERGI & 4.76 & 5.71 & 5.32 & 5.37 & 5.32 & 6.38 & 5.61 \\
QSTRUC & 3.20 & 2.99 & 3.19 & 3.05 & 2.94 & 2.90 & 2.89 \\
\hline
\end{tabular}

Table 3.30: Execution times of various phases for COMMIX-1AR/P code running on an Alliant $\mathrm{FX} / 80$ for P1r2.

\begin{tabular}{||l|r|r|r|r|r|r|r||}
\hline Phase & SV & SC & SVC & SVC & SVC & SVC & SVC \\
8ce & 1ce & 4ce & 4ce & 8cc \\
\hline Time step & 12297.20 & 9986.37 & 12358.54 & 11450.86 & 10351.53 & 10433.30 & 10019.23 \\
Momentum constr. & 3733.70 & 3110.72 & 3579.02 & 3554.11 & 3212.87 & 3299.41 & 3133.59 \\
Momentum solu. & 4071.72 & 2904.95 & 4233.12 & 3471.16 & 3074.20 & 3007.06 & 2967.60 \\
Energy constr. & 3888.48 & 3461.57 & 3831.89 & 3881.43 & 3578.85 & 3656.77 & 3458.13 \\
Energy solu. & 497.44 & 406.23 & 609.70 & 440.80 & 383.85 & 367.56 & 358.76 \\
Thermal Struct. & 21.59 & 21.79 & 21.79 & 21.98 & 22.02 & 22.00 & 22.02 \\
TS Radiation & 1.90 & 1.93 & 1.83 & 1.84 & 1.82 & 1.91 & 1.77 \\
\hline
\end{tabular}

Table 3.31: Execution times of CPU intensive routines in COMMIX-1AR/P code running on an Alliant FX/80 for P1r2.

\begin{tabular}{||l|r|r|r|r|r|r|r||}
\hline Routine & $\begin{array}{r}\text { SV } \\
\text { 1ce }\end{array}$ & $\begin{array}{r}\text { SC } \\
\text { 8ce }\end{array}$ & $\begin{array}{r}\text { SVC } \\
\text { 1ce }\end{array}$ & $\begin{array}{r}\text { SVC } \\
\text { 2ce }\end{array}$ & $\begin{array}{r}\text { SVC } \\
\text { 4ce }\end{array}$ & $\begin{array}{r}\text { SVC } \\
\text { 6ce }\end{array}$ & $\begin{array}{r}\text { SVC } \\
\text { 8ce }\end{array}$ \\
\hline SOLVEV & 458.38 & 375.29 & 570.36 & 410.00 & 357.43 & 341.73 & 334.15 \\
LOWFCV & & 907.13 & & & & & 934.60 \\
QSTDRS & 999.55 & 886.96 & 959.58 & 982.58 & 952.28 & 960.68 & 906.94 \\
XMOMI & 435.81 & 359.01 & 393.95 & 373.60 & 315.14 & 328.59 & 311.66 \\
YMOMI & 587.24 & 399.98 & 519.84 & 503.95 & 432.57 & 442.40 & 428.66 \\
ZMOMI & 679.56 & 556.98 & 682.26 & 705.87 & 666.21 & 700.76 & 641.23 \\
ENERGI & 685.20 & 586.70 & 734.43 & 771.56 & 663.17 & 694.49 & 659.76 \\
QSTRUC & 421.05 & 386.95 & 419.25 & 295.88 & 382.98 & 379.64 & 377.24 \\
\hline
\end{tabular}


Table 3.32: Routine events for COMMIX-1AR/P trace graph obtained from the Cray X-MP/48.

\begin{tabular}{|l|l||l|l||l|l||l|l|}
\hline Event & Routine & Event & Routine & Event & Routine & Event & Routine \\
\hline \hline 0 & COMMIX & 1 & LOCF & 2 & CLEAR & 3 & TSCAN \\
4 & MXPLNS & 5 & ALTER & 6 & AMAIN & 7 & GEOM 3D \\
8 & BOXES & 9 & FILLM & 10 & SHOME & 11 & TLEFTS \\
12 & INITIAL & 13 & INITA3 & 14 & INITA2 & 15 & FITIT \\
16 & SMOOTH & 17 & GETF & 18 & INHTX & 19 & INPUMP \\
20 & INFORC & 21 & INPSTR & 22 & ICTEMP & 23 & BARIN \\
24 & RSET2 & 25 & DSET2 & 26 & REDEF & 27 & BCTEMT \\
28 & BCTEM0 & 29 & BCTEMP & 30 & BCFLOW & 31 & BCPRES \\
32 & GETMTS & 33 & GETIJK * & 34 & LODODD & 35 & HSTRUC \\
36 & HEATCF & 37 & TSTRUC & 38 & QSTRUC & 39 & OUTPUT \\
40 & PSTRU1 & 41 & RARRAY & 42 & PSTRUC & 43 & GDCONV \\
44 & WATSTP & 45 & GETEKL & 46 & TIMSTP & 47 & MOLOOP \\
48 & XMOMI & 49 & YMOMI & 50 & ZMOMI & 51 & FORCES \\
52 & PUMPQ & 53 & PEQN & 54 & CGLOOP & 55 & STORE \\
56 & UPDATE & 57 & COMMAT & 58 & BOUND & 59 & ELIM \\
60 & VOLCEL & 61 & WRPTST & 62 & SORTC & 63 & RESORT \\
64 & CNGRIC & 65 & FACNCV & 66 & COFSRT & 67 & OPERXDF \\
68 & LOWFCV $*$ & 69 & DDOTC * & 70 & DAXPYC & 71 & DAXPXC \\
72 & MOMENI & 73 & ENLOOP & 74 & ESORCE & 75 & ENERGI \\
76 & SOLVEV & 77 & QSRAD & 78 & WATTIM & 79 & RESTAR \\
80 & PLTAPE & & & & & & \\
\hline
\end{tabular}

\subsubsection{Dynamic program execution tracing analysis}

As mentioned in Section 2.3 we have started using tools developed at CSRD to capture the detailed histories of routine invocation together with machine performance statistics. In Figure 3.1, we show the routine trace graph for a COMMIX-1AR/P execution in vector mode on the Cray X-MP. Only fifty iterations were performed in this execution. Each routine has been given an event number as listed in Table 3.32. ${ }^{1}$ The trace graph visually depicts where time is being spent in routines during the execution and the routine calling dynamics as the application proceeds. We hope to further apply this type of analysis in Phase 2 to identify performance limiting behavior.

\subsection{Results from COMMIX-1C}

We next list some results from the performance evaluation of COMMIX-1C on the ANL Cray XMP/14 and the Alliant FX/8. For this particular code we also include performance results from running the code in its original environment, namely a Sun Sparc workstation. We note that although to be consistent with our previous runs we used the SV version of the code as baseline, COMMIX-1C was not written to profit from vector processing.

\footnotetext{
${ }^{1}$ Some of the routine events have been elided due to their high frequency; these events are identified by an "*n in the Table 3.32 .
} 


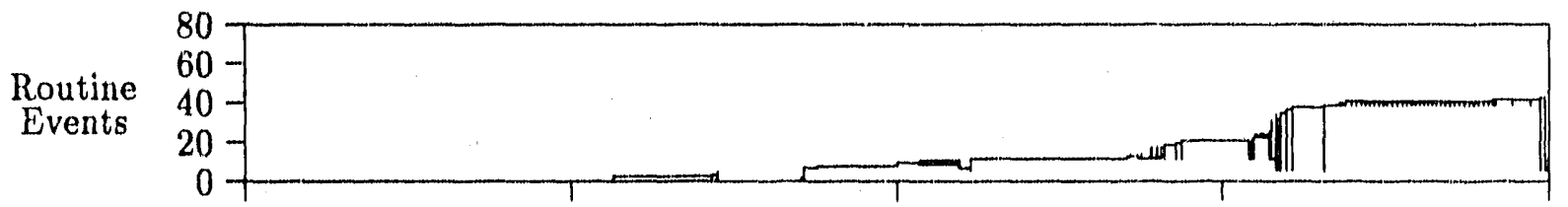

Ist,

bighlitl

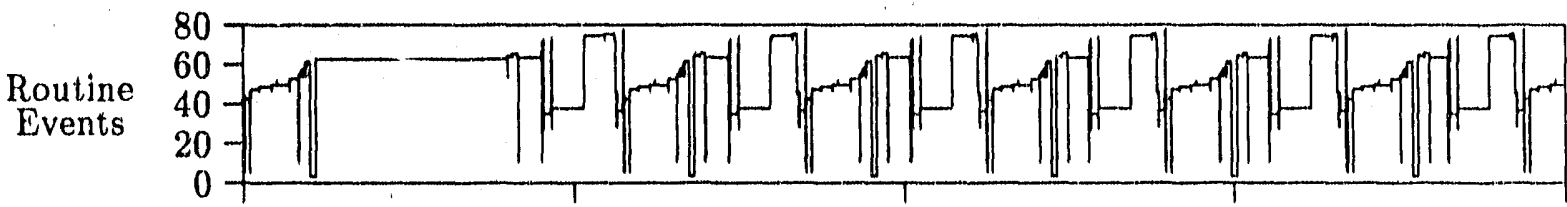

2nd lïglitlı

Routine

Events

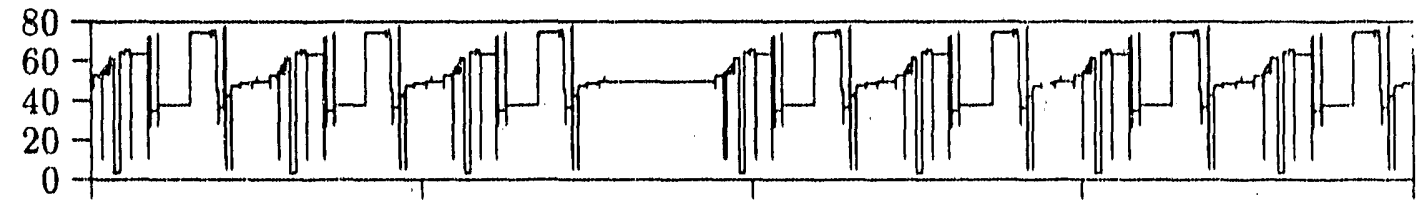

$3 r d$ lightill

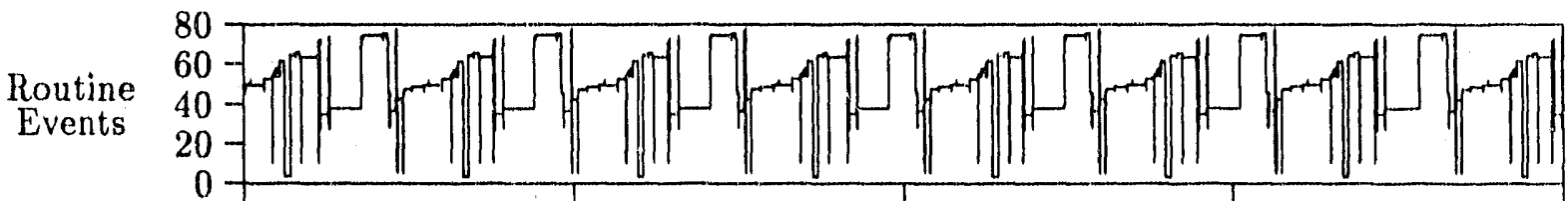

4 ll lighth

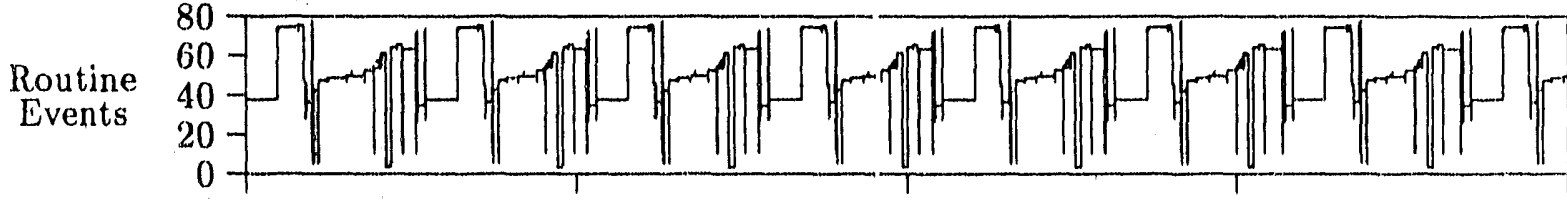

\section{$5 t h$} lighlith

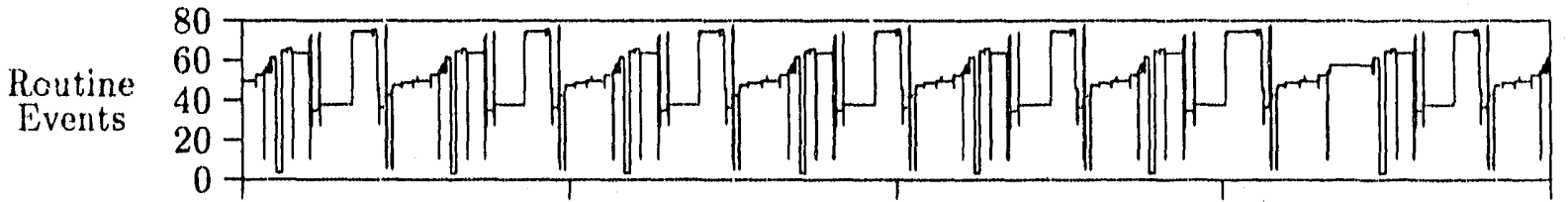

(j). righth

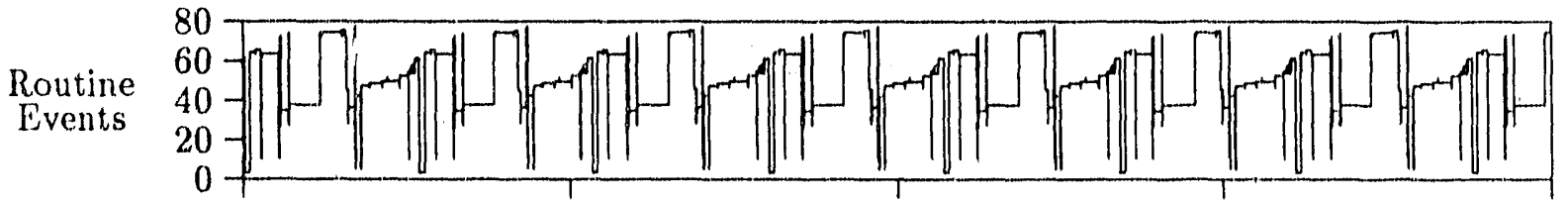

7 th bightil

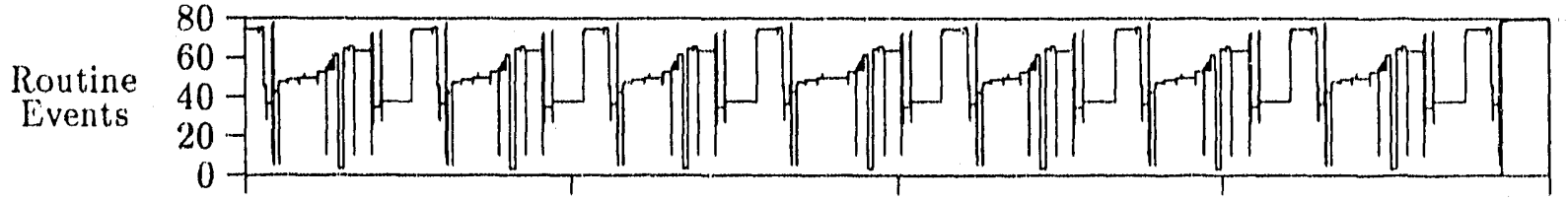

81,1 riglilt

Figure 3.1: Routine graph for fifty iterations of COMMIX-1AR/P execution on the Cray X-MP/48. 
Table 3.33: Timing results from COMMIX-1C runs on the Sparc, Cray X-MP/14 and Alliant FX/8.

\begin{tabular}{||l|c|c|c||}
\hline & C1r0 & C1r1 & C1r2 \\
\hline Sparc & 40.3 & $9,233.4$ & $3,512.5$ \\
Cray (SV) & 6.6 & $1,463.6$ & 554.8 \\
Alliant (SVC) & 48.4 & $12,625.9$ & $5,124.1$ \\
\hline
\end{tabular}

Table 3.34: HPM group 0 summary for baseline SV COMMIX-1C code running on Cray X-MP/14 with data set C1r2.

\begin{tabular}{||l|r||}
\hline & C1r2 \\
\hline CPU seconds & 554.86 \\
Million inst/sec (MIPS) & 36.28 \\
Avg. clock periods/inst & 3.24 \\
\% CP holding issue & 45.67 \\
Inst.buffer fetches/sec & $0.61 \mathrm{M}$ \\
Floating adds/sec & $2.30 \mathrm{M}$ \\
Floating multiplies/sec & $2.93 \mathrm{M}$ \\
Floating reciprocal/sec & $0.38 \mathrm{M}$ \\
l/O mem. references/sec & $0.49 \mathrm{M}$ \\
CPU mem. references/sec & $12.18 \mathrm{M}$ \\
Floating ops/CPU second & $5.60 \mathrm{M}$ \\
\hline
\end{tabular}

To port the code from the Sparc to the Cray X-MP, we first had to change data type declarations to Cray single precision (64 bits), IEEE arithmetic traps specific to the Sun were removed, machine specific constants were changed, and dynamic memory allocation calls were converted to Cray specific. Several difficulties associated with a "Namelist" like input processor were also resolved. For the Alliant the above mentioned IEEE arithmetric traps were converted to Alliant traps. However, the principal problem was the lack of compatible dynamic memory allocation system routines (calls to the Alliant Fortran allocate() were not enough.) The problem was dealt with (for the present) by explicit allocation of large sections of memory, coded so as to check that the allocation is sufficiently large. In addition the use of a parallel/vector architecture led to concerns about the correctness of the computed results. This led us to perform additional experiments with various compiler options and run on a single processor. These experiments revealed that our transformations did not change the nature of the output data.

The results from Table 3.33 imply that the MFLOPS rates achieved for phase 2 of the computation were 0.88 on the Sparc, 5.6 on the Cray X-MP/14 and 0.61 on the Alliant FX/8.

We also list the HPM results from running the code in SV mode on the ANL Cray X-MP/14 and using data set C1r2. Tables 3.34-3.37 summarize the obtained results.

We see that the current version of the code performs very badly on the Alliant: Despite the automatic nntimizations, the times are lower for the Alliant compared to the Sparc. The inability to automat $y$ extract high performance for this code was also observed from the speedup values: Input deck $\mathrm{C} 1 \mathrm{r} 2$ ran only 1.03 times faster on 8 than on a single CE of the FX/8. 
Table 3.35: HPM group 1 summary for baseline SV COMMIX-1C code running on Cray X-MP/14 with data set C1r2.

\begin{tabular}{||r|r||}
\hline & C1r2 \\
\hline Waiting on A-registers/funct. units & 13.28 \\
Waiting on S-registers/funct. units & 29.63 \\
Waiting on V-registers & 0.23 \\
Waiting on vector functional units & 0.36 \\
Waiting on scaler memory references & 0.18 \\
Waiting on block memory references & 1.37 \\
\hline
\end{tabular}

Table 3.36: HPM group 2 summary for baseline SV COMMIX-1C code running on Cray X-MP/14 with data set $\mathrm{C} 1 \mathrm{r} 2$.

\begin{tabular}{||l|r||}
\hline & C1r2 \\
\hline Inst. buffer fetches/sec & $0.61 \mathrm{M}$ \\
Scalar memory refs/sec & $7.77 \mathrm{M}$ \\
\% having - nflicts & 36.14 \\
I/O memory refs/sec & $0.64 \mathrm{M}$ \\
\% having conflicts & 51.72 \\
Block memory refs/sec & $4.41 \mathrm{M}$ \\
\% having conflicts & 72.96 \\
Vector memory ref $3 / \mathrm{sec}$ & $0.72 \mathrm{M}$ \\
\hline
\end{tabular}

Table 3.37: HPM group 3 summary for baseline SV COMMIX-1C code running on Cray X-MP/14 with data set $\mathrm{C} 1 \mathrm{r} 2$.

\begin{tabular}{|c|c|c|}
\hline \multirow[b]{2}{*}{ type of instruction } & \multicolumn{2}{|c|}{ Clr2 } \\
\hline & instr./CPU sec & $\%$ of all inst. \\
\hline jump/special & $3.30 \mathrm{M}$ & 9.11 \\
\hline scalar & $32.85 \mathrm{M}$ & 90.54 \\
\hline witor integer/logical & $0.05 \mathrm{M}$ & 0.15 \\
\hline vector floating point & $0.02 \mathrm{M}$ & 0.04 \\
\hline vetor memory & $0.06 \mathrm{M}$ & 0.16 \\
\hline type of operation & ops/CPU sec. & avg. VL \\
\hline Vector integer\&logical & $0.57 \mathrm{M}$ & $10 \overline{60}$ \\
\hline Vector floating point & $0.25 \mathrm{M}$ & 16.45 \\
\hline Vector memory & $0.72 \mathrm{M}$ & 12.19 \\
\hline
\end{tabular}


Looking at the HPM group 3 results in Table 3.37, we notice that the vector floating-point operations accounted for only $0.04 \%$ of all instructions. This compares with a typlcal $0.5 \%$ for the corresponding baseline SV runs of $1 \mathrm{AR} / \mathrm{P}$ and $0.6 \%$ for the $\mathrm{SV}(\mathrm{Zv})$ runs. This low performanco $1 \mathrm{~s}$ not surprising: COMMIX $1 \mathrm{C}$ uses YSMP to solve the linear systerns by direct methods which do not take advantage of vector or parallel processing capabilities. Finally, a comrnent similar to that made in the end of Section 3.2.2 regarding vector lengths and inefficiency of SVC processing could also be made here. 


\section{Chapter 4}

\section{WHAMS-3D description}

The WHAMS-3D computer program employs explicit time integration to do nonlinear, transient analysis of frames, shells, plates and continua in three dimensions [4]. Both material nonlinearlties due to elasto-plastic behavi or and geometric nonlinearities due to large displacements can be treated. This program has been developed jolntly at Northwestern University and Argonne National Laboratory and is Internationally recognized as a state-of-the-art program for performing nonllnear transient analysis. WHAMS-3D has maintained its role as a leading edge program by performing extensive research in innovative methods (e.g., subcycling) for enhancing computational efficiency. Argonne has employed and developed this program to perform analysis of various reactor: components and structures in a computationally efficlent manner. Other organizations have employed the program to perform analysis of ice forces on Arctic structures, analysis of buried structures, military weapon analysis, aircraft engine structural analysis, analysis of electronic com. ponents, reactor safety fluid-structural analysis, impact and penetration analysis, and automobile crashworthiness simliations. The program has been extensively validated by comparisons with a wide range of experiments associated with large deformations, buckling, impact-penetration, etc.

The program employs a finite element format, so that it possesses considerable versatility in modeling complex shapes and boundary conditions. The element library consists of the following: quadrilateral and triangular plate-shell elements, a beam element, a spring element and a hexahedral continuum element. In addition, a rigid linkage is included which permits the efficient modeling of very stiff portions of a structure, such as the bottom ring of a core barrel. In a rigid linkage, the motion of a master node defines the motion of all slave nodes linked to the master node. 'This option is also useful fo" eccentrically connected elements where the midlines of the connected elements do not coincide, as for example, in stiffeners.

All of the elements in the program are three dimensional. The beam element is based on Euler Bernoulli theory, which assumes that planes normal to the midline remain planar and normal. The element has stiffness in torsion, bending about two axes, and in the axial direction.

If the material response is elasto-plastic, the cross-section of the beam is restricted to be thinwalled, and the cross-section is completely arbitrary and defined through input. Each olemont is assumed to be prismatic. For elastic beams, the cross-section may be defined directly through the section moduli and transverse shears may be included.

The quadrilateral plate element is based on Mindlin-Relssner theory. This is the recommended element for most simulations. It uses one-point quadrature in the surface of the shell to achieve computational efficiency. Spurious modes are suppressed through a consistent gamma-projection. A Mindlin-Reissner type triangular plate element is also available. The triangular element provides 
versatility in modeling.

A three dimensional Lagrangian hexahedral elemont with elght nodes ls lncluded which can be used to model fludd and solid continua. The eloment uses only ond quadrature point with a consistent control of hourglass modes so very large meshes can be handlod effectively.

In all of the elements, a corotational olement formulation ls used, In this formulation a coor". dinate system is embedded in each element and all element computations are performed relative to this element coordinate system which rotates with the element. This introduces considerable simplifications into the formulation and adds substantially to the efficiency of the program.

Time integration is performed by the exphicit, central difference method. Stable thme steps can be automatically computed or input by the user or a driving program. Mixed thme integration, $a$ procedure which allows for different time stepes in different parts of the mesh, may be employed. To provide a check on the stability of a calculation after it is completed, energy balances are computed. A lumped mass matrix is employed so that no matrix inversion is needed in the computatlons and core s orage requirements are minimized.

El isto-plastic materlal laws using Mises or llyushin criterlon with lsotropic strain hardening are Included. Hardening is defined by a plecewise linear function. Elastic and liydrodynamic material laws are also available. The material laws are completely modularized, so other material laws can easily be added by the user.

An interaction algorithm for treating impact-penetration simulations with arbitrary crosion is included. This algorithm is based on interaction between slave nodes (projectile) and master elements (target) and no tracking of the sliding interfaces is needed. It employs an assombly of normals to identify the interface surface adaptively so that it can handle eroding elements in both the target and penetrator. In the interaction algorithm, momentum is exchanged betweon interacting nodes so that the total momentum is conserved.

The algorithm for explicit time integration primarily involves computing the internal forces of the elements which describe the simulation. Nodal accelerations, velocities and displacements are then made by central difference equations.

\subsection{Data sets}

Four data sets are used for the performance testing. These data sets all use quadrilateral plate elements. They, however, represent four different physical problems.

Cylindrical Panel (cylpanel). Since closed form solutions are not available for nonlinear transient programs, solutions obtained by finite elements are typlcally compared to experimental results. The cylindrical panel problem has been used as a benchmark for many nonlinear transient programs. Experimental results have been obtained for this shell by Balmor and Witner [3]. A. 120 degree cylindrical panel subjected to an impulsive loading is modeled to test the elastic-plastic, large deformation capability and the ability to treat curvod surfaces. The analytical model takes advantage of symmetry, so only half the panel is modeled; 1089 nodes and 1024 elements were used for the half panel. The impulsive loading is accomplished numerically by prescribing an initial velocity.

Column Buckling (buckle). In the Clinch River breeder reactor design, four columns were used to support the above core structures. The dynamic buckling analysis quantifies the energy absorption capability of these columus during core disruptive accidents. Symmetry conditions 
Table 4.1: Executlon times for WHAMS-3D on the Cray X-MP/48 (from IIPM).

\begin{tabular}{||r|r|r|r|r||}
\hline Version & \multicolumn{4}{|c|}{ Exacution Tlmo } \\
\cline { 2 - 5 } & buckle & cylpand & framo & spcap \\
\hline S & 69.19 & 301.19 & 4048.66 & 88.02 \\
SV & 9.73 & 64.11 & 650.07 & 14.72 \\
SVC & 9.66 & 61.63 & 633.92 & 15.30 \\
\hline
\end{tabular}

allow a half column model of 287 nodes and 240 elements. The column is loaded hy prescribing an upward velocity of $500 \mathrm{in}$. $/$ sec. to the bottom nodes of the mesh with the top nodes fixud.

Spherical Cap (spcap). The pressure loaded spherical cap problem is a common benchmark for nonlinear finite element codes. Linear elastic and elastic-plastlc materials are typically con. sidered. A total of 332 nodes and 300 elements were used for the one-quarter model. A uniform pressure loading is applied over the cap.

Structural Frame (frame). The frame mesh is a common frontend automoblle structural component subjected to crashworthiness testing. Hallquist, Benson, and Goudreau have documented the geometry definitlons of thls data set which was obtained from Suzuki Motor Company. of Japan and used to benchmark the performance of other finite element formulations for shell analysis [7]. A total of 1122 nodes and 1100 elements were used for the mesh in sur benchmark analyses. Impact was modeled by prescribing a uniform velocity of $800 \mathrm{in} . / \mathrm{sec}$. across the mesh with a clamped row of nodes at one end.

\subsection{Results from WHAMS 3D}

A detailed analysis of our timing results and porting activities can be found in the first progress report. We outline here the performance measurements we have obtained during the flrst phase of this project. In this Initial benchmark we tried to get all the performance results based on original code. In particular, we did not attempt any hand optimizations.

As mentioned in previous sections, the orjginal code WIIAMS3I) has been ported, compiled, and tested on both the Cray X-MP/48 of NCSA and the Alliant FX/80 of CSRD. On the Clay $\mathrm{X}-\mathrm{MP} / 48$, we obtain three different (scalar, scalar-vector, and scalar-vector-concurrent optimized) versions of the object codes. On the Alliant FX/80, we have four different (scalar, scaldr-vertor, scalar-concurrent, and scalar-vector-concurrent optimined) versions. In this section, we present some of the performance results of these versions of the original code on both machines for all four data sets.

\subsubsection{Cray X-MP/48}

The performance results of the original code WIIAMS3D on the Cray X-MP/48 are presented in Tables 4.1 through 4.5. Table 4.1 shows the execution time for all four data sets. As can be obtained from this table, the vector speedup of the SV version over the S version ranges from 5.98 for the data set spcap to 6.23 for frame. It is also observed that the SVC version does not yield good 
Table 4.2: Execution times for SVC version of WHAMS-3D on the Cray X.MP/48 (from IIPM).

\begin{tabular}{||c|r|r|r|r||}
\hline \multirow{2}{*}{$\begin{array}{c}\text { No. of } \\
\text { Concurrent } \\
\text { CPUs }(i)\end{array}$} & \multicolumn{4}{|c||}{$\begin{array}{c}\text { Connect tirne }\left(T_{i}\right) \\
\text { in each CPU }\end{array}$} \\
\cline { 2 - 5 } & buckle & cylpanel & frame & spcap \\
\hline 1 & 3.15 & 19.66 & 162.35 & 5.99 \\
2 & 0.59 & 9.92 & 131.54 & 1.88 \\
3 & 2.45 & 12.30 & 147.89 & 4.34 \\
4 & 3.47 & 19.74 & 211.34 & 3.08 \\
\hline Total exec. time & 9.66 & 61.63 & 653.12 & 15.30 \\
Total CPU time & 25.54 & 155.37 & 1714.46 & 35.10 \\
\hline
\end{tabular}

Table 4.3: Floating point operations on Cray X-MP/48 for the S version (from group 0 of IIPM).

\begin{tabular}{|l|r|r|r|r|}
\hline F.P. type & \multicolumn{4}{|c|}{ No. of F.P. operations } \\
\cline { 2 - 5 } & buckle & cylpanel & frame & spcap \\
\hline adds & $290.8 \mathrm{M}$ & $2,021.7 \mathrm{M}$ & $19,954.2 \mathrm{M}$ & $430.8 \mathrm{M}$ \\
multiplies & $345.6 \mathrm{M}$ & $2,254.0 \mathrm{M}$ & $23,588.5 \mathrm{M}$ & $504.3 \mathrm{M}$ \\
reciprocals & $36.0 \mathrm{M}$ & $203.9 \mathrm{M}$ & $2,473.2 \mathrm{M}$ & $55.0 \mathrm{M}$ \\
\hline Total & $672.4 \mathrm{M}$ & $4,479.6 \mathrm{M}$ & $46,0,15.9 \mathrm{M}$ & $990.1 \mathrm{M}$ \\
\hline
\end{tabular}

Table 4.4: MFLOPS for WHAMS-3D on the Cray X-MP/48 (from uPM).

\begin{tabular}{||c|r|r|r|r||}
\hline \multirow{2}{*}{ Version } & \multicolumn{4}{|c||}{ MFLOPS } \\
\cline { 2 - 5 } & buckle & cylpanel & frame & spcap \\
\hline S & 11.36 & 11.50 & 11.37 & 11.25 \\
SV & 71.00 & 71.65 & 72.65 & 69.05 \\
SVC & 71.38 & 74.49 & 76.97 & 66.56 \\
\hline
\end{tabular}


Table 4.5: Performance data for WHAMS-3D on the Cray X-MP/48 using data set cylpanel (from FLOWTRACE).

\begin{tabular}{||l|r|r|r|r|r|r|r||}
\hline Compiler optlons & \multicolumn{2}{|c|}{ S } & \multicolumn{2}{|c|}{ SV } & \multicolumn{2}{|c|}{ SVC } \\
\hline name & calls & $\%$ time & time & \% time & time & \% time & time \\
\hline QPLATE & 25600 & 33.16 & 1.29 .00 & 28.52 & 18.30 & 28.69 & 17.62 \\
QMISES & 12800 & 20.97 & 81.56 & 17.56 & 11.27 & 17.55 & 10.78 \\
QRIGID & 25600 & 9.08 & 35.33 & 7.04 & 4.52 & 7.00 & 4.30 \\
QFORCE & 25600 & 6.13 & 23.83 & 16.29 & 10.46 & 16.68 & 10.25 \\
UPDATE & 1600 & 5.48 & 21.33 & 3.69 & 2.37 & 3.69 & 2.27 \\
SOLVE & 1 & 5.33 & 20.73 & 6.36 & 4.08 & 6.29 & 3.87 \\
BYIELV & 63568 & 5.28 & 20.55 & 3.94 & 2.53 & 3.97 & 2.44 \\
NEWSDV & 63568 & 4.35 & 16.93 & 4.53 & 2.91 & 4.56 & 2.80 \\
QVECTV & 25600 & 3.53 & 13.75 & 2.24 & 1.44 & 2.29 & 1.41 \\
PMODV & 63568 & 2.83 & 11.01 & 2.58 & 1.66 & 2.58 & 1.59 \\
CONSTR & 1600 & 1.87 & 7.26 & 1.86 & 1.20 & 1.88 & 1.16 \\
\hline TOtal & $-\cdots-$ & 98.01 & 381.28 & 94.61 & 60.74 & 95.18 & 58.49 \\
\hline
\end{tabular}

performance although four CPUs are available. Table 4.3 shows the MFLOPS for the $S$ version of the code on the Cray X-MP/48, as obtained from group 0 of HPM.

Table 4.2 gives the job accounting information for the SVC version. This information providus the connect time in each active CPU and the number of active CPUs (cf. the discussion Eq. 3.1). The performance of the scalar-vector version of this code reaches about $70 \mathrm{MFLOPS}, 1 / 3$ of the peak performance of this machine.

To better understand the behavior of this code, we also used the performance utllity FLOWTRACE to gather information at the subroutine level. All the four data sets have been examined. In the following, we present only the performance data for the data set cylpanel since the belavior of this code on the other three data sets does not vary very much. The results are shown in Table 4.5 in which only heavily used subroutines are presented. A brief description of these most timeconsuming subroutines is given below.

QPLATE Compute the internal forces for a quadrilateral plate element using one point integration by a velocity strain formulation.

QMISES Compute the stress given the strain for a plane stress biaxial elastic-plastic material.

QRIGID Compute deformed nndal coordinates for a quadrilateral plate element, calculate the values of the shape functions at integration point $(0,0)$, and calculate the area of the element.

QFORCE Compute hourglass forces, transfer forces from element to global coordinate system, and update the global internal force vector.

UPDA'TE Update the nodal coordinate system.

SOLVE Integrate the equations of motion $-F=$ ma.

BYIELDV Bring back stress to yield surface. 
Table 4.6: Execution time for WHAMS-3D on the Alliant FX/80 (from GPRof).

\begin{tabular}{||l|c|c|c|c|c||}
\hline Optimization & No. of & \multicolumn{5}{|c||}{ Execution Time } \\
\cline { 3 - 6 } & CEs & buckle & cylpanel & frame & spcap \\
\hline S & 1 & 541.63 & 3802.19 & 37951.01 & 820.16 \\
SV & 1 & 231.62 & 1595.33 & 15811.41 & 328.20 \\
\hline
\end{tabular}

Table 4.7: Exerution time for the SC version of WHAMS-3D on the Alliant FX/80 (from GPROF).

\begin{tabular}{||c|c|l|l|l||}
\hline \multirow{2}{*}{$\begin{array}{c}\text { No. of } \\
\text { CEs }\end{array}$} & \multicolumn{4}{|c||}{ Execution Time } \\
\cline { 2 - 5 } & buckle & cylpanel & frame & spcap \\
\hline 1 & 568.63 & 3997.18 & 40759.16 & 815.39 \\
2 & 339.89 & 2368.70 & 23685.83 & 478.80 \\
4 & 215.77 & 1508.11 & 14643.84 & 302.38 \\
8 & 155.82 & 1060.39 & 10346.51 & 228.20 \\
\hline
\end{tabular}

NEWSDV Compute new stress by elastic-plastic material law.

QVECTV Compute the quadrilateral plate element coordinate system.

PMODV Look up the plastic modulus and yield stress.

CONSTR Enforce boundary conditions in the local coordinate system.

\subsubsection{Alliant FX/80}

We next present results from use of the Alliant FX/80. It should be noted that we did not use the compiler option $-\mathrm{r} 8$ to compile the source code. In other words, all versions on the Alliant are in single precision.

Table 4.6 shows the execution time using only one CE for the S and SV options. The vector speedup of the SV version over the S version ranges from 2.34 to 2.50 , which is reasonably good. It should be noted that the timings for the $S$ and $S V$ versions presented were obtained without

Table 4.8: Execution times for the SVC version of WHAMS-3D on the Alliant FX/80 (from GPROF).

\begin{tabular}{||c|c|c|c|c||}
\hline \multirow{2}{*}{$\begin{array}{c}\text { No. of } \\
\text { CEs }\end{array}$} & \multicolumn{4}{|c||}{ Execution Time } \\
\cline { 2 - 5 } & buckle & cylpanel & frame & spcap \\
\hline 1 & 289.50 & 1896.92 & 19916.66 & 407.77 \\
2 & 257.04 & 1763.51 & 17301.31 & 357.67 \\
4 & 196.99 & 1347.01 & 12812.99 & 289.34 \\
8 & 166.45 & 1094.76 & 11054.41 & 236.21 \\
\hline
\end{tabular}


Table 4.9: MFLOPS for WHAMS-3D on the Alliant FX/80.

\begin{tabular}{||l|c|c|c|c|c||}
\hline Optimization & No. of & \multicolumn{5}{|c||}{ MFLOPS } \\
\cline { 3 - 6 } & CFs & buckle & cylpanel & frame & spcap \\
\hline S & 1 & 1.24 & 1.21 & 1.21 & 1.24 \\
SV & 1 & 2.90 & 2.88 & 2.91 & 3.10 \\
SC & 8 & 4.32 & 4.33 & 4.45 & 4.45 \\
SVC & 8 & 4.04 & 4.19 & 4.16 & 4.30 \\
\hline
\end{tabular}

Table 4.10: Performance data for WHAMS-3D on the Alliant FX/80 using data set cylpanel (from GPROF).

\begin{tabular}{||l|r|r|r|r|r|r|r||}
\hline \multicolumn{2}{|c|}{ Version } & \multicolumn{2}{c|}{ S } & \multicolumn{3}{c|}{ SV } & \multicolumn{2}{c||}{ SVC } \\
\hline No. of CE's used & \multicolumn{2}{|c|}{1} & \multicolumn{2}{|c|}{1} & \multicolumn{2}{|c|}{8} \\
\hline name & calls & \%time & time & \%time & time & \%time & time \\
\hline QPLATE & 25600 & 38.2 & 1452.83 & 25.2 & 401.33 & 15.7 & 171.75 \\
QMISES & 12800 & 14.3 & 544.90 & 19.8 & 316.36 & 26.2 & 287.13 \\
QRIGID & 25600 & 10.6 & 404.41 & 7.5 & 119.16 & 4.3 & 46.16 \\
QFORCE & 25600 & 6.6 & 251.00 & 9.1 & 145.91 & 10.2 & 112.18 \\
UPDATE & 1600 & 4.6 & 173.54 & 4.2 & 67.21 & 6.0 & 65.60 \\
SOLVE & 1 & 8.0 & 304.90 & 14.6 & 232.82 & 20.5 & 224.67 \\
BYIELV & 63568 & 3.2 & 123.40 & 2.7 & 43.54 & 2.2 & 24.21 \\
NEWSDV & 63568 & 5.2 & 197.44 & 4.7 & 75.31 & 2.5 & 27.67 \\
QVECTV & 25600 & 2.1 & 80.21 & 1.8 & 29.09 & 1.3 & 14.10 \\
PMODV & 63568 & 2.1 & 79.86 & 1.7 & 27.40 & 1.7 & 18.40 \\
CONSTR & 1600 & 2.6 & 98.80 & 3.5 & 55.52 & 3.3 & 35.59 \\
\hline Total & --- & 97.5 & 3711.29 & 94.8 & 1513.65 & 93.9 & 1027.91 \\
\hline
\end{tabular}

explicitly specifying to use only one CE. The effect of the explicit specification of using one CE on performance is currently under investigation. Tables 4.7 and 4.8 show the execution time using 1 , 2,4 , and $8 \mathrm{CEs}$ for the SC and SVC versions, respectively. As can be seen from these two tables, the parallelism obtained through the use of compiler options results in speedups across processors for the SVC version that are rather poor.

Table 4.9 compares the performances in terms of MFLOYS for different versions of the object code, where we have used the floating point operation counts of the $S$ version obtained from the Cray X-MP/48 because the Alliant does not have utility to gather this information.

Table 4.10 shows the performance data at the subroutine level using the Alliant FX/80 for data set cylpanel for the S, SV, and SVC versions of the object code. Note that the time is reduced by a factor of 2.45 in going from the $S$ to the SV version on one processor. However, going to SVC decreases the time only by a factor of 1.47 , giving a multiprocessor efficiency of only $18 \%$. It should also be observed from 4.7 and 4.8 that the SVC version did not yield better performance than the $\mathrm{SC}$ version when all eight CE's were used. This is due to the fact that the vector length is not long 
Table 4.11: Characteristics of the data sets used in WHAMS-3D.

\begin{tabular}{||l|rr||}
\hline Data Set & \multicolumn{2}{|c||}{ Problem Size } \\
& No. of nodes & No. of time steps \\
\hline buckle & 287 & 800 \\
cylpanel & 1089 & 1600 \\
frame & 1122 & 16000 \\
spcap & 332 & 1000 \\
stcn & 407 & 1136 \\
\hline
\end{tabular}

Table 4.12: Execution times for WHAMS-3D on the ANL Alliant FX/8.

\begin{tabular}{|c|c|c|c|c|c|c|}
\hline \multirow[t]{2}{*}{ Data Set } & \multicolumn{2}{|c|}{$\begin{array}{l}\text { Scalar Mode } \\
\text { (fortran }-0 g \text { ) }\end{array}$} & \multicolumn{2}{|c|}{$\begin{array}{c}\text { Vector Mode } \\
\text { (fortran - Ogv) }\end{array}$} & \multicolumn{2}{|c|}{$\begin{array}{l}\text { Vector-Conc. Mode } \\
\text { (fortran -Ogve) }\end{array}$} \\
\hline & user & system & user & system & user & system \\
\hline & 691.1 & 1.1 & 336.4 & 1.1 & 181.1 & 1.1 \\
\hline & 4419.2 & 1.1 & 2286.4 & 1.7 & 1212.1 & 1.5 \\
\hline frame & 44654.6 & 1.3 & 23030.0 & 1.6 & 12343.5 & 1.5 \\
\hline spcap & 940.8 & 1.0 & 492.0 & 1.1 & 277.3 & 1.0 \\
\hline stcn & 604.4 & 0.9 & 324.0 & 1.1 & 173.9 & 1.1 \\
\hline
\end{tabular}

enough to take advantage of both vectorization and parallelization, as explained for the COMMIX code.

\subsubsection{Additional Results}

In addition to the Cray X-MP/48 of NCSA and the Alliant FX/80 of CSRD, we have also run the baseline code on the Argonne ARCF Alliant FX/8, Cray X-MP/14, and Sparcstation. Data sets employed in the test on these three machines include the four data sets mentioned previously and an additional one: a steel containment (stcn) subjected to a pressure loading. The problem sizes of these data sets are listed in Table 4.11. The performance data are presented in Tables 4.12 through 4.14 . Table 4.12 shows the timings on the Alliant FX/8 using scalar optimization, vectorization, and parallelization. Table 4.13 presents the execution time for the Sparcstation and the Cray X-MP/14. A summary of the findings of this assessment on the Cray is provided in Table 4.14.

As indicated by the very high ratio of vector floating point operations to total floating point operations in Table 4.14, it is clear that this code has been vectorized to a large degree. The average vector lengths are close to the optimal vector length (64). A further indicator of good vectorization is the relatively low hold-issue condition percentage for the scalar registers and functional units as opposed to the vector registers and functional units. For maximum performance, the highest percentage of hold-issues should be on the vector registers and vector functional units as these are the fastest. The columns for hold issue conditions indicate the number of clock periods during which the issuance of an instruction was (held) delayed. Note that more than one hold issue condition 
Table 4.13: Execution times for WHAMS-3D on the Sparc, Cray X-MP/14, and Alliant FX/8.

\begin{tabular}{||l|r|r|r|r|r||}
\hline \multirow{2}{*}{ Machine } & \multicolumn{5}{|c||}{ Execution Time } \\
\cline { 2 - 6 } & buckle & cylpanel & frame & spcap & stcn \\
\hline scalar Sparcstation & 896.9 & 6173.1 & 61647.9 & 1322.1 & 857.4 \\
Cray X-MP/14 (vector) & 10.3 & 67.1 & 689.7 & 8.4 & 11.5 \\
\hline
\end{tabular}

Table 4.14: HPM performance data summary for WHAMS-3D running on the Cray X-MP/14.

\begin{tabular}{|c|c|c|c|c|c|c|c|c|c|}
\hline \multirow[t]{3}{*}{$\begin{array}{l}\text { Data } \\
\text { Set }\end{array}$} & \multicolumn{2}{|c|}{$\begin{array}{c}\text { Floating Point } \\
\text { Operations }\end{array}$} & \multicolumn{5}{|c|}{$\begin{array}{c}\text { Hold Issue } \\
\text { Conditions (\%) }\end{array}$} & \multicolumn{2}{|c|}{ MFLOPS } \\
\hline & $\%$ Vectorized & Average VL & & & & tor & Block & Total & Vector \\
\hline & & & A-rg. & S-rg. & rg. & f.u. & & & \\
\hline buckle & 99 & 58.78 & 7.1 & 8.3 & 27.3 & 25.4 & 27.5 & 67.2 & 66.7 \\
\hline cylpanel & 99 & 63.19 & 6.0 & 9.0 & 27.1 & 25.7 & 31.8 & 68.4 & 67.7 \\
\hline frame & 99 & 60.63 & 6.2 & 8.9 & 28.2 & 26.1 & 31.6 & 68.5 & 67.7 \\
\hline spcap & 96 & 58.76 & 6.9 & 18.1 & 24.7 & 15.8 & 25.3 & 47.3 & 45.4 \\
\hline stcn & 100 & 59.75 & 7.5 & 11.6 & 24.4 & 21.6 & 27.7 & 56.0 & 55.0 \\
\hline
\end{tabular}


can occur during a given clock period so that the individual percentages cannot be added to get the overall percentage. Removing hold issue conditions promises to offer code speedups however the compiler attempts to catch bottlenecks and even the most optimized codes may have large percentages for hold issues. Only after further experimentation can it be determined whether the number of hold issues conditions can be reduced.

\subsubsection{Dynamic program execution tracing analysis}

In Figure 4.1, we show the routine trace graph for the first 2.954625 seconds of the WHAMS-3D execution in vector mode on the Cray X-MP; eight consecutive sub-sections of this interval are shown. Each routine has been given an event number as listed in Table 4.15. ${ }^{1}$ The trace graph visually depicts where time is being spent in routines during the execution and the routine calling dynamics as the application proceeds. We hope to further apply this type of analysis in Phase 2 to identify performance limiting behavior.

\footnotetext{
${ }^{1}$ Some of the routine events have been elided due to their high frequency; these events are identified by an "*" in the Table 4.15.
}

\begin{tabular}{|l|l||l|l||l|l||l|l|}
\hline Event & Routine & Event & Routine & Event & Routine & Event & Routine \\
\hline \hline 0 & MAIN & 1 & DRIVE & 2 & READKO & 3 & COREE \\
4 & READMA & 5 & READNE & 6 & DECOD & 7 & CROSS \\
8 & BLOCKS & 9 & ASSBLE & 10 & QASME & 11 & QVECTR \\
12 & QDELT & 13 & READLD & 14 & READOU & 15 & SOLVE \\
16 & OUTPUT & 17 & LOADPR * & 18 & FRCIN & 19 & QFRCIN \\
20 & QNODE & 21 & QVECTV * & 22 & QRIGID * & 23 & QPLATE \\
24 & QMISES * & 25 & QFORCE * & 26 & PMODV * & 27 & BYIELV * \\
28 & NEWSDV * & 29 & CONSTR & 30 & UPDATE & 31 & ETIME2 \\
32 & BTIME & 33 & ROUTP'T & 34 & PLOTER & & \\
\hline
\end{tabular}

Table 4.15: Routine events for WHAMS-3D trace graph 


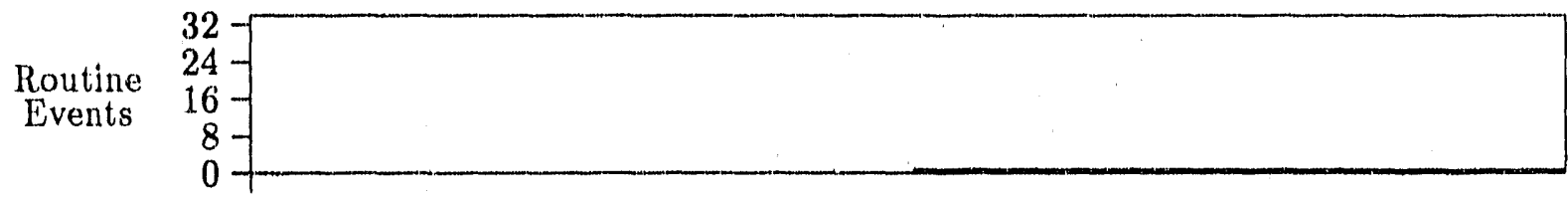

1 st

Eghth

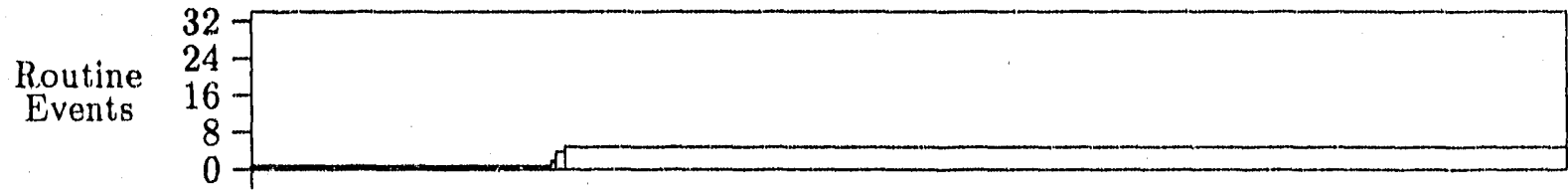

2nd

righth

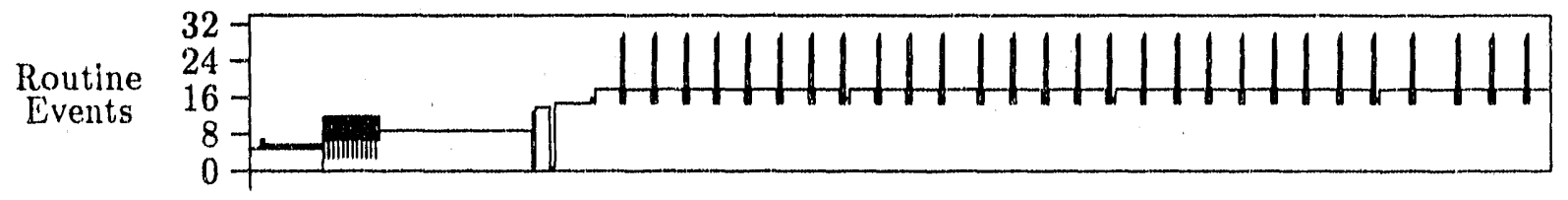

$3 r d$ Bighth

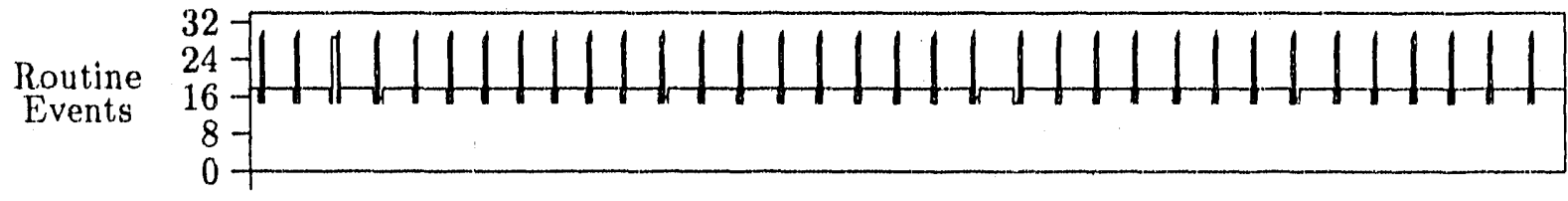

4th lighth

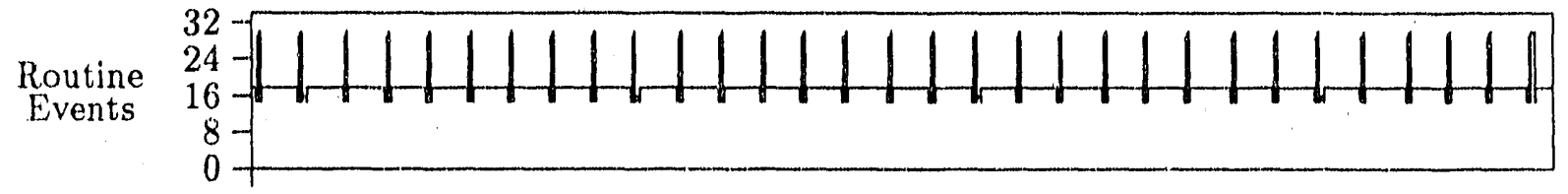

5 th Eightl

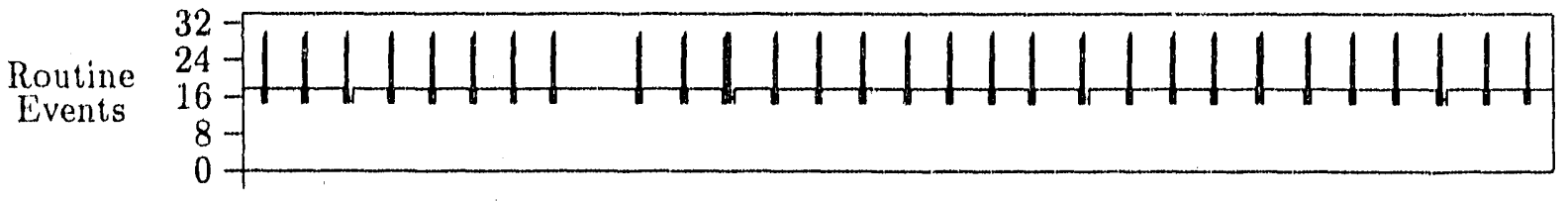

6tl Eighth

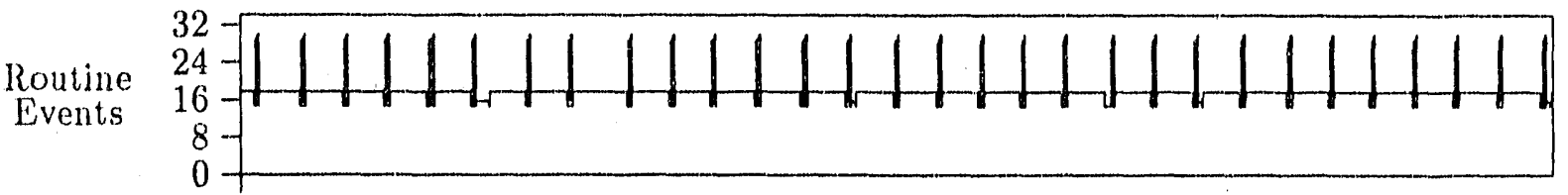

7 th righth

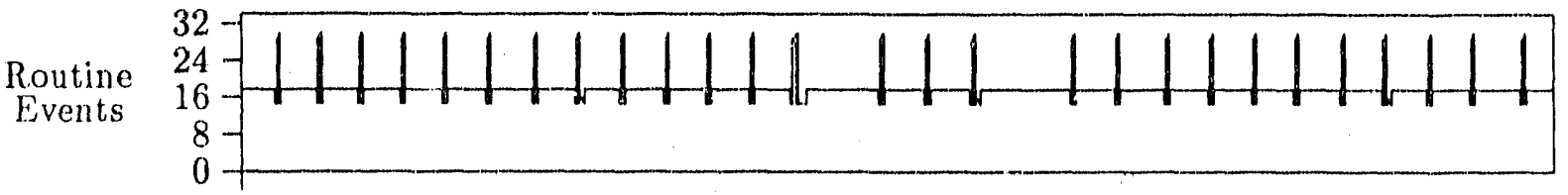

8th Eiglith

Figure 4.1: Routine graph for 0.0 to 2.954625 seconds of the WHAMS-3D execution 


\section{Chapter 5}

\section{Conclusion}

We have completed the work prescribed for Phase 1 of our project. We have performed a large number of experiments without any hand tuning of the COMMIX-1AR/P, COMMIX-1C and WHAMS3D codes. Our purpose was to have yardsticks by means of which future improvements to the code could be measured. Nevertheless, we were also able to obtain a lot of data for different compilation options, as part of our Phase 2 efforts.

Although, a full appreciation of the results requires time, even at this stage, several interesting; observations can be made, which were not apparent before our experiments had taken place. These observations can be found in the commentary for the tables in the previous Sections.

We found that the performance of the COMMIX codes on the Cray and Alliant is much below the acceptable range, meaning that the codes have to be modified to take advantage of the parallel and vector processing aspects of these architectures. We noticed that even for COMMIX-1AR/P, whose parts dealing with the linear system solvers had been vectorized, the performance is very low and will remain such, until the parts of the code dealing with the matrix assembly are also modified. In that spirit we started testing the standard automatic vectorization/parallelization tools available on the target machines. Our experiments indicate the following: Without any help from the user, the tools only provide slight performance gains. Some help in the form of inline directives to the compiler can introduce very significant improvements in the performance of some subroutines. These experiences point to the next phase of our work, namely examining the individual subroutines to provide help to the automatic restructuring tools, and using more advanced data dependence tests to recover computations which can be processed in parallel.

For WHAMS-3D we have seen that the performance of the Cray under vector processing was quite reasonable. Nevertheless, the performance under the multiprocessing options was not satisfactory. In the next phase of this project we intend to investigate further the automatic transformation tools coupled with user directives to produce better optimized code. 


\section{Bibliography}

[1] Analytical Thermal and Hydraulic Research Program, Components Tecinol. ogy Division, Argonne National laboratory, Commixu1B: A threedimensional transient single-phase computer program for thermal hydraulic analysis of single and multicomponent. systems. Volume I: Equations and Numerics, Sep. 1985.

[2] - Commix-1B: A three-dimensional transient single-phase computer program for thermal hydraulic analysis of single and multicomponent systems. Volume II: User's Manual, Sep. 1985.

[3] H. A. BALMER AND E. A. WITMER, Theoretical-experimental correlation of large dynamic and permanent deformation of impulsively loaded simple structures, Tech. Rep. FDP-TDR-64-108, Wright Patterson AFB, Ohio, 1964.

[4] T. Belytschko and C. S. Tsay, WHAMSE: A program for three-dimensional nonlinear structural dynamics, Tech. Rep. NP.2250, Dept. Civil Engin., Northwestern University, Evanston, Illinois, Feb. 1982. Research Project 1065-3.

[5] R. N. Blomquist, P. Garner, and E. M. Gelbard, Code abstract for COMMIX-1AR/P, July 1989.

[6] Cray Research, Inc., UNICOS Performance Utilities Reference Manual, May 1989.

17] J. O. Hallquist, D. J. Benson, And G. L. Goudreau, Implementation of a modified Hughes-Liu shell into a fully vectorized explicit finite element code, in Finite Elements For Nonlinear Problems, P. G. Bergan, K. J. Bathe, and W. Wunderlicl, eds., Springer-Verlag, Berlin, 1986, pp. 465-479.

[8] R. Hockney, $\left(r_{\infty}, n_{1 / 2}, s_{1 / 2}\right)$ measurements on the 2-CPU Cray X-MP, Parallel Computing, 2 (1985), pp. 1-14.

[9] A. Malony, J. LaRson, AND D. REeD, Tracing applicaition program execution on the Cray $X-M P$ and Cray 2, Tech. Rep. 985, Center for Supercomputing Research and Development, Nov. 1990. 


\section{Appendix A}

Appendix: Milestones for FY 1991

Copies of this Appendix can be obtained from the authors. 

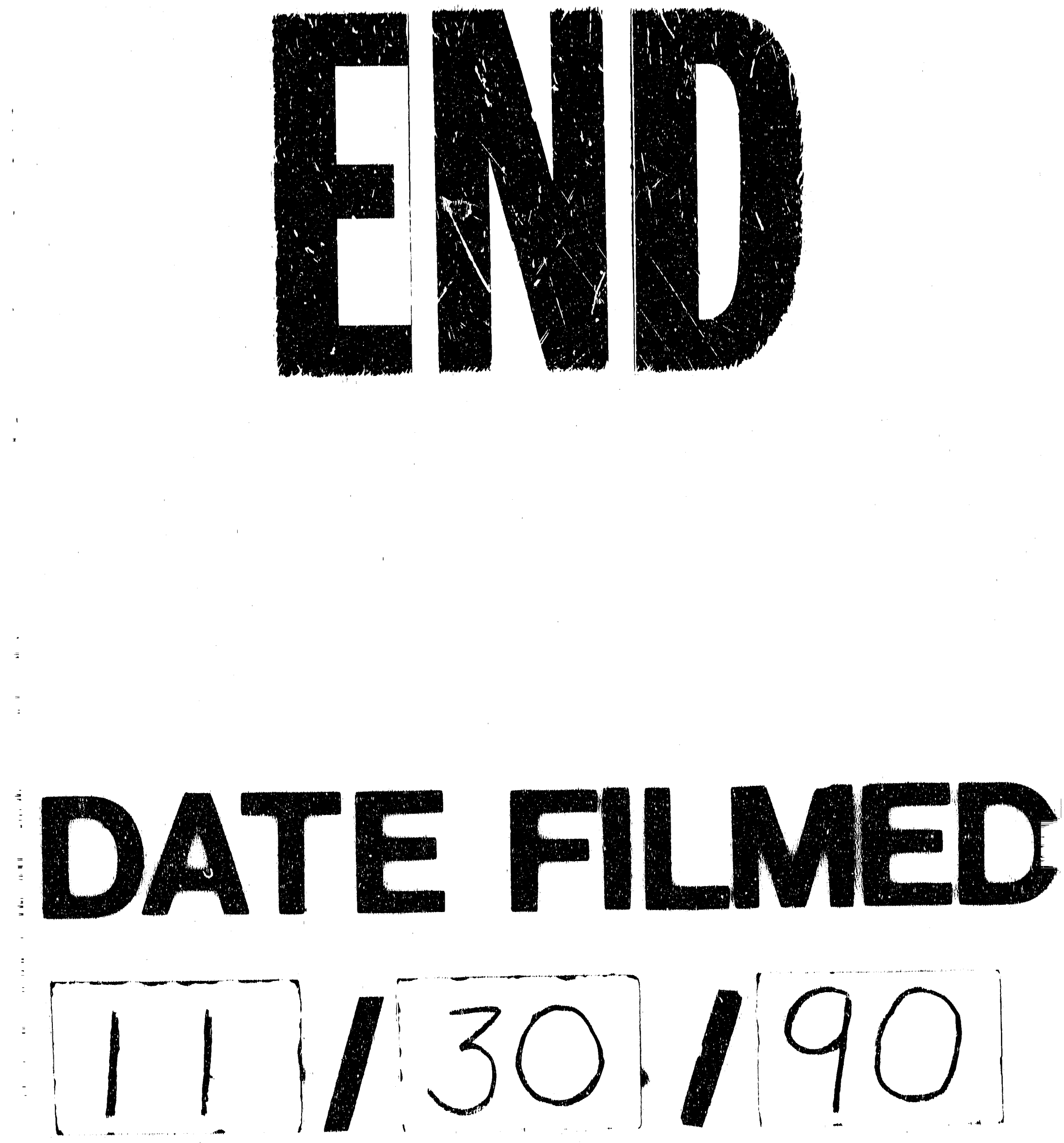
